\title{
12. CENOZOIC SILICOFLAGELLATES FROM ROCKALL PLATEAU, DEEP SEA DRILLING PROJECT LEG 81 ${ }^{1}$
}

\author{
David Bukry, U.S. Geological Survey, Scripps Institution of Oceanography, La Jolla ${ }^{2}$
}

\begin{abstract}
Eocene to Pliocene silicoflagellates from the Rockall Plateau are sparse to moderately abundant and include assemblages at the upper and lower boundaries of the Miocene Series. Relative paleotemperature values for silicoflagellates from the upper Miocene and lower Pliocene at DSDP Hole 552A based on quantitative data are cooler than are those from equatorial Pacific Sites 503 and 504, but show a matching warm peak at the Miocene/Pliocene boundary and a major cooling in the lower Pliocene.

Five new taxa of silicoflagellates are defined from Rockall Plateau assemblages: Corbisema panda Bukry, n. sp., Dictyocha pulchella var. inflata Bukry, n. var., Distephanus xenus Bukry, n. sp., Mesocena apiculata evexa Bukry, n. subsp., and Naviculopsis pacifica pansa Bukry, n. subsp.
\end{abstract}

\section{INTRODUCTION}

Cenozoic silicoflagellates occur in 34 of 66 samples processed from DSDP Leg 81 at Rockall Plateau in the North Atlantic. The best silicoflagellate assemblages bracket the Miocene/Pliocene boundary at Deep Sea Drilling Project (DSDP) Hole 552A and provide a relative paleotemperature curve for Cores 11 to 30 , which correlates with the curves for tropical eastern Pacific upwelling areas at DSDP Holes 503A and 504. The similarity in paleotemperature trends between these assemblages indicates an oceanwide response, instead of local upwelling patterns. Even though the occurrence patterns of Distephanus jimlingii (Bukry) and D. speculum minutus (Bachmann) are similar between North Atlantic DSDP Holes 552A and 408, the coeval assemblages at DSDP Site 408 have distinctly lower relative paleotemperature values. The first Atlantic occurrences of two newly described, short-ranged, Pacific silicoflagellates-Dictyocha concinna Bukry and D. neonautica Bukry-are noted in Hole 552A in their expected sequence.

Other silicoflagellate occurrences for Leg 81 include: early and middle Eocene at DSDP Hole 553A, late Miocene at DSDP Holes 554 and 554A, and middle and late Miocene at DSDP Hole 555. DSDP Leg 48, which cored the Rockall Plateau in July of 1976, obtained silicoflagellates from Site 403 (lower Miocene) just east of Site 553 and from Site 406 (upper Eocene, upper Oligocene, and lower Miocene) south of the Leg 81 sites (Fig. 1); these are reported for the first time.

The zonation applied to the Rockall Plateau silicoflagellate assemblages is from Bukry (1981b and in press). Although some cosmopolitan or low-latitude zonal guide species, such as Dictyocha angulata Bukry, are missing, many others, such as Corbisema triacantha (Ehrenberg), Dictyocha hexacantha Schulz, D. neonautica Bukry, D. pul-

\footnotetext{
${ }^{1}$ Roberts, D. G., Schnitker, D., et al., Init. Repts. DSDP, 81: Washington (U.S. Govt. Printing Office). 92093.
}

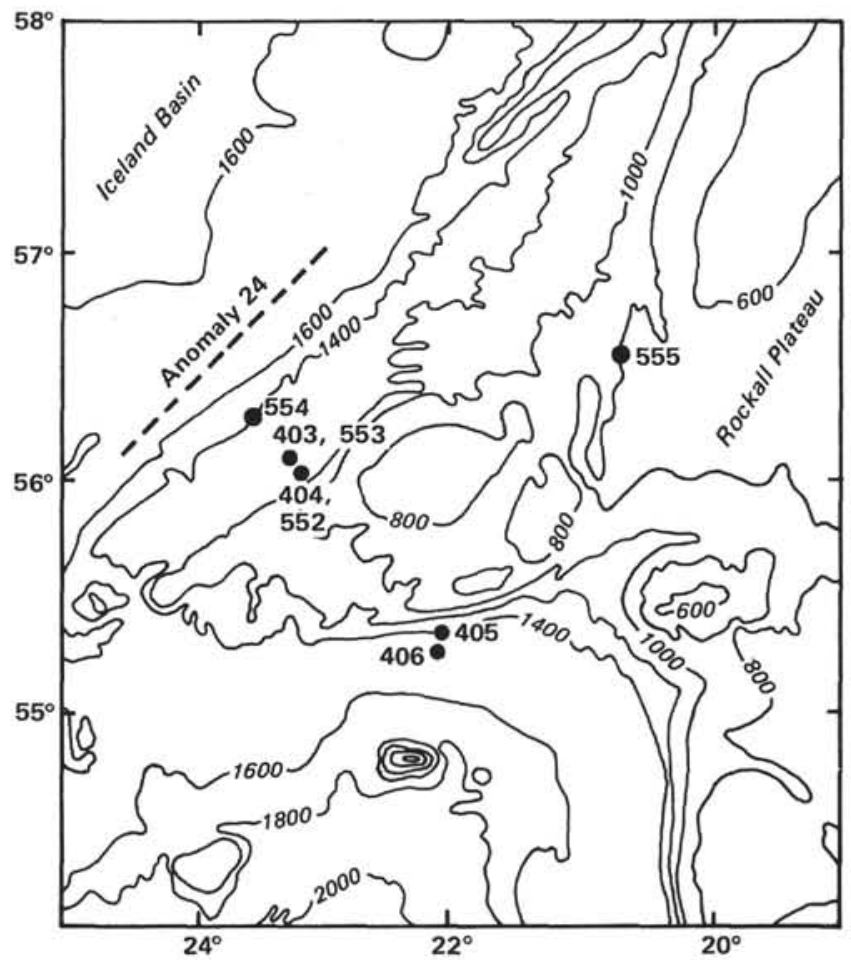

Figure 1. Location of Deep Sea Drilling Project Leg 81 Sites 552 to 555 and Leg 48 Sites 403 to 406 at Rockall Plateau, west of Scotland. Depths contoured in fathoms.

chella Bukry, Distephanus speculum haliomma (Ehrenberg), D. stauracanthus (Ehrenberg), Naviculopsis biapiculata (Lemmermann), and $N$. foliacea Deflandre, do permit recognition of low- to middle-latitude zones (Fig. 2). The assemblages lack the restricted cold-water species described from more northerly areas of the Atlantic (Perch-Nielsen, 1976; Bukry, 1976b; and Martini and Müller, 1976).

\section{RELATIVE PALEOTEMPERATURE VALUES}

The relative paleotemperature values $(T s)$ calculated from generic and morphological abundance data of sili- 


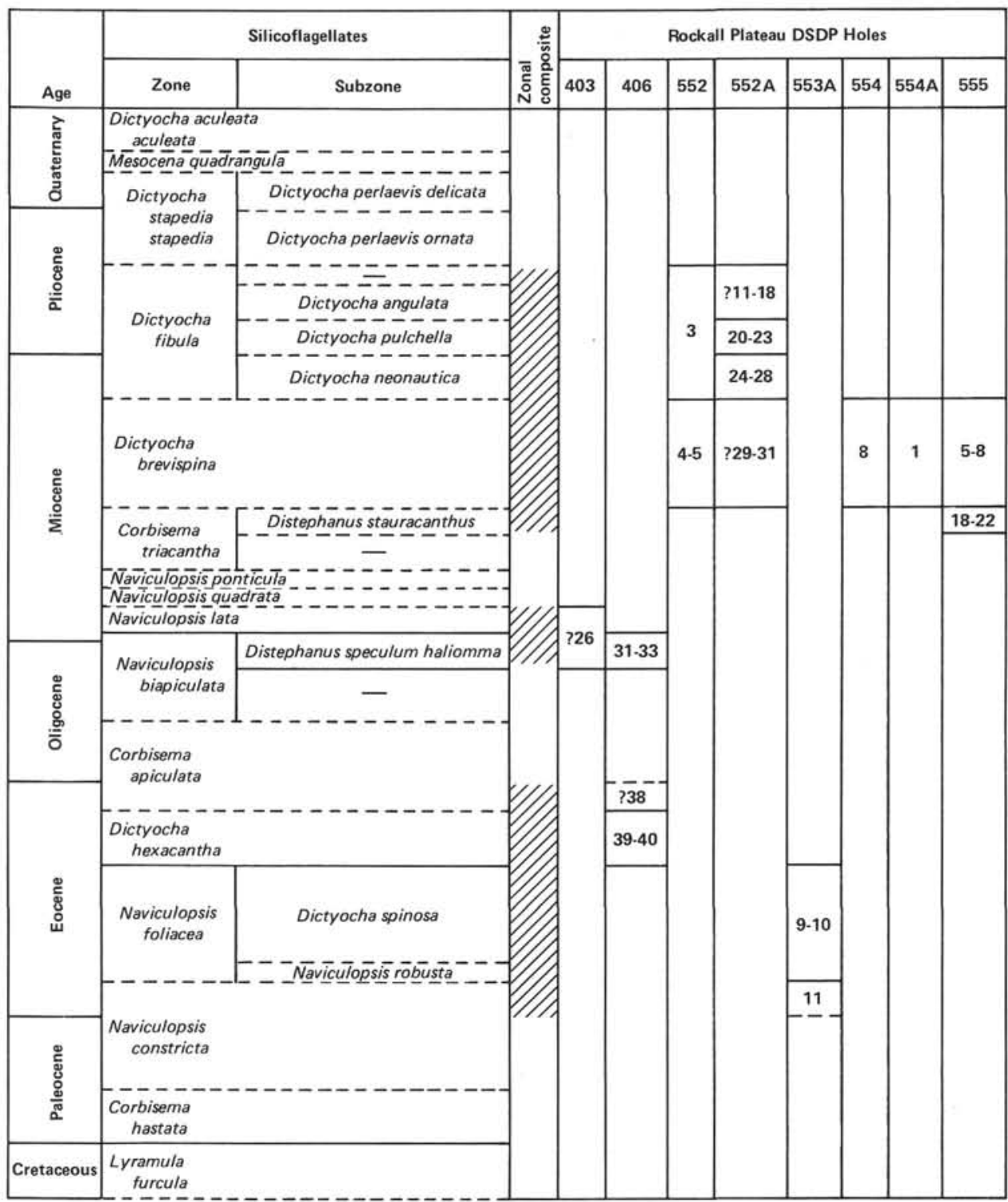

Figure 2. Zonal and geologic age assignment of DSDP cores from Rockall Plateau in the North Atlantic, as indicated by silicoflagellates in examined samples. The range of zones identified from Leg 48 and Leg 81 is shown by cross-hatching.

coflagellates (Bukry, 1981a, and in press) have been determined and plotted for Hole 552A of Leg 81 (Fig. 3). The shape of the curve across the Miocene/Pliocene boundary has the same trends as Holes 503A and 504 from the eastern equatorial Pacific. A general warming trend for the uppermost Miocene is punctuated by a minor cooling just below the Miocene/Pliocene boundary. The culmination of this warming trend just above the boundary is followed by a strong decline in paleotemperature values to about $4.1 \mathrm{Ma}$ in Holes $503 \mathrm{~A}$ and 504 and about 4.0 to $4.5 \mathrm{Ma}$ in Hole 552A, in which D. pulchella disappears at all three sites. The following warm peak at about 3.4 to $3.8 \mathrm{Ma}$ gives higher $T s$ values than the Miocene/Pliocene boundary warm peak at all three sites. The agreement between relative paleotemperature trends in the Atlantic and Pacific oceans shows that fluctuations in silicoflagellate paleotemperature indicators reflect oceanwide events, not local upwelling.
The Ts numeric values in Hole 552A for the Miocene/Pliocene boundary warm peak have a maximum of $T s=66$, which is lower than the $T s=86$ in Hole $503 \mathrm{~A}$ and the $T s=85$ in Hole 504. Similarly the lower Pliocene cool trough is cooler $(T S=30)$ at the higher latitude Hole 552A than at the equatorial Hole 503A $(T s=68)$ and Hole $504(T s=42)$. Even though the $T s$ values show the difference in latitude between Leg 81 and equatorial Pacific assemblages, the broad paleotemperature trends for the oceanic photic zone were quite similar during the latest Miocene and early Pliocene in these areas.

\section{CORONATID DISTEPHANUS FLUCTUATIONS}

The occurrence of coronatid specimens (six distal pikes on apical ring) of both Distephanus speculum minutus and, to a greater degree, $D$. speculum speculum in Hole 552A permits a comparison to relative paleotemperature values 


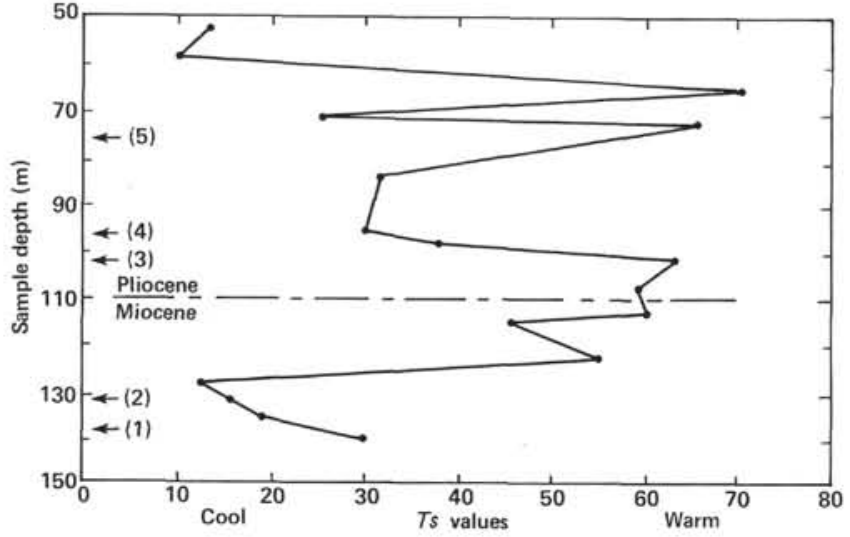

Figure 3. Silicoflagellate relative paleotemperature values $(T S)$ plotted for core-sample depths below the seafloor at DSDP Hole 552A. The curve shows a warm peak at the Miocene/Pliocene boundary (approx. $110 \mathrm{~m}$ ) which declines into a cool trough in the lower Pliocene (between 83 and $98 \mathrm{~m}$ ). This pattern also occurs in the equatorial Pacific at DSDP Holes 503A and 504 for coeval assemblages. Five diatom reference events (Baldauf, this volume) are shown for correlation. See Table 3 for silicoflagellate reference events. Diatom events: (1) first Thalassiosira praeconvexa. (2) first T. convexa, (3) last T. miocenica, (4) first $T$. oestrupii, and (5) first Nitzschia jouseae.

(Ts) (Table 1) to determine whether the accessory structure has any correlation to paleotemperature. The proportion of specimens of Distephanus with apical pikes fluctuates through Cores 11 to 30 in Hole 552A, with the ratios of normal to coronatid specimens ranging from 0.1 to 75.0 . There is no consistent pattern of fluctuations that matches changes in $T s$. Equally low normal/coronatid ratio values of 0.9 to 0.7 in Cores 14 and 15 correspond to a high $T s=$

Table 1. Ratios of normal to coronatid specimens of Distephanus fluctuate independently from relative paleotemperature values $(T s)$ for Miocene and Pliocene strata at DSDP Hole 552A. Coronatid data from Table 3 .

\begin{tabular}{|c|c|c|c|c|}
\hline $\begin{array}{c}\text { Sample } \\
\text { (depth in } \mathrm{cm} \text { ) }\end{array}$ & $\begin{array}{l}\text { D. speculum } \\
\text { minutus } \\
\text { ratio: } \\
\text { normal/ } \\
\text { coronatid }\end{array}$ & $\begin{array}{l}\text { D. speculum } \\
\text { speculum } \\
\text { ratio: } \\
\text { normal/ } \\
\text { coronatid }\end{array}$ & $\begin{array}{c}\text { Total } \\
\text { ratio: } \\
\text { normal/ } \\
\text { coronatid }\end{array}$ & $T s$ \\
\hline \multicolumn{5}{|l|}{ Hole 552A } \\
\hline $11-3,145-146$ & 1.0 & 75.0 & 38.0 & 13 \\
\hline $12-3,135-136$ & 0.5 & 2.9 & 2.6 & 10 \\
\hline $14-3,110-111$ & 0.0 & 0.9 & 0.9 & 71 \\
\hline $15-3,110-111$ & 0.1 & 0.8 & 0.7 & 27 \\
\hline $16-1,136-137$ & 0.5 & 3.0 & 2.4 & 66 \\
\hline $18-1,95-97$ & 0.0 & 1.9 & 1.9 & 32 \\
\hline $20-2,95-97$ & $<0.5$ & 5.3 & 1.2 & 30 \\
\hline $21-2,104-106$ & 4.0 & 3.5 & 3.6 & 38 \\
\hline $22-2,104-106$ & 2.8 & 7.0 & 4.2 & 63 \\
\hline $23-3,98-99$ & 1.0 & 2.4 & 2.3 & 59 \\
\hline $24-3,98-99$ & 8.0 & 18.0 & 17.0 & 60 \\
\hline $25-2,67-68$ & 0.1 & 3.0 & 1.0 & 45 \\
\hline $26-3,92-93$ & 18.0 & 16.0 & 34.0 & 55 \\
\hline $27-2,110-111$ & $<40.0$ & 4.8 & 9.9 & 12 \\
\hline $28-2,120-121$ & 1.0 & 10.0 & 3.2 & 16 \\
\hline $29-3,60-61$ & $<0.3$ & 7.0 & 4.7 & 19 \\
\hline $30-2,36-37$ & $<5.0$ & 14.5 & 16.0 & 30 \\
\hline
\end{tabular}

71 in Core 14 and a low $T s=27$ in Core 15. Also, Cores 23 and 24 have nearly equal $T S$ values (59 and 60, respectively), but the normal/coronatid ratios of 2.3 and 17.0 are widely disparate. The abundance of coronatid specimens of Distephanus is not a function of relative paleotemperature in the Leg 81 cores. Distephanus speculum var. bispicatus, cruxoid Distephanus mesophthalmus, and D. crux bispinosus developed apical pikes in the late Miocene, which may have been a structural adaptation to increased upwelling or predation, a relation previously suggested by Kitchell (1979) for certain Paleogene specimens with elongate basalring spines. Elongate specimens of Dictyocha, such as $D$. neonautica, $D$. transenna, elongate variants of $D$. brevispina, and very long-spined specimens of the Distephanus speculum group, also occur in the late Miocene, supporting the upwelling hypothesis. Production of coronatid specimens might have been a seasonal effect, leading to larger randomness in the abundance values for coronatid specimens.

\section{SITE 552}

\section{$\left(5^{\circ} 02.56\right.$ ' $\mathrm{N}, 2^{\circ}{ }^{\circ} 13.39$ ' W, depth $\left.2311-2315 \mathrm{~m}\right)$}

Site 552 is just oceanward of the southwest corner of Hatton-Edoras Bank. Miocene and Pliocene silicoflagellates were studied from Cores 3 to 5 and $11 \mathrm{~A}$ to $31 \mathrm{~A}$, recovered between 53 and $145 \mathrm{~m}$ sub-bottom (Tables 2, 3 ). The assemblages are common to abundant and diverse, with both warm- and cool-water taxa present. A new succession of Miocene to Pliocene zones and subzones from the equatorial eastern Pacific (Bukry, in press) can be recognized, in part, at Site 552, suggesting a warm-water connection between the Pacific and Atlantic through a central American seaway.

The Dictyocha fibula Zone/D. brevispina Zone boundary is tentatively drawn below Sample 552A-28-2, 120-121 $\mathrm{cm}(131 \mathrm{~m})$ because of the lowest occurrence of auxiliary guide species Dictyocha longa var. paxilla Bukry, which is related to the new zonal guide species $D$. longa (Bukry, in press). The presence of Dictyocha neonautica Bukry in higher samples in Cores 552A-24-3 and 27 (112 to $117 \mathrm{~m}$ ) supports this boundary location. The old zonal criterion, the asperoid/fibuloid $(\mathrm{A} / \mathrm{F})$ dominance reversal in genus Dictyocha, occurs much higher in the lower Pliocene between Cores 16 and 18 ( 73 to $83 \mathrm{~m}$ ). But the original Hole 552 shows an isolated fibuloid dominance much lower in Core $5(129 \mathrm{~m})$. Although several short-term reversals in A/F dominance are tabulated for Site 503, the broad biogeographic pattern of dominance has shown that fibuloid taxa became dominant earlier, in the late Miocene, at low latitudes, but achieved dominance only later in the early Pliocene at mid latitudes, Sites 310 and 464 (Bukry, 1982a), similar to that observed in Site 552 .

The lower Pliocene or upper Miocene Dictyocha pulchella Subzone and upper Miocene Dictyocha neonauti$c a$ Subzone are identified by their name-giving boundary species for Hole 552A, showing the same stratigraphic succession as recorded in DSDP Holes 503A and 504 in the eastern equatorial Pacific Ocean. Another similarity with the Pacific for Hole 552A is the corresponding paleoceano- 
Table 2. Late Miocene silicoflagellate biostratigraphy and relative paleotemperature values (TS) for Cores 3 to 6 of DSDP Hole 552. Abundances shown as percents. Presence of opal phytoliths noted by $X$. Preparations from Core-Sections 12-2, 12-6, 13-1, 14-2, 16-1, and 18-1 are barren.

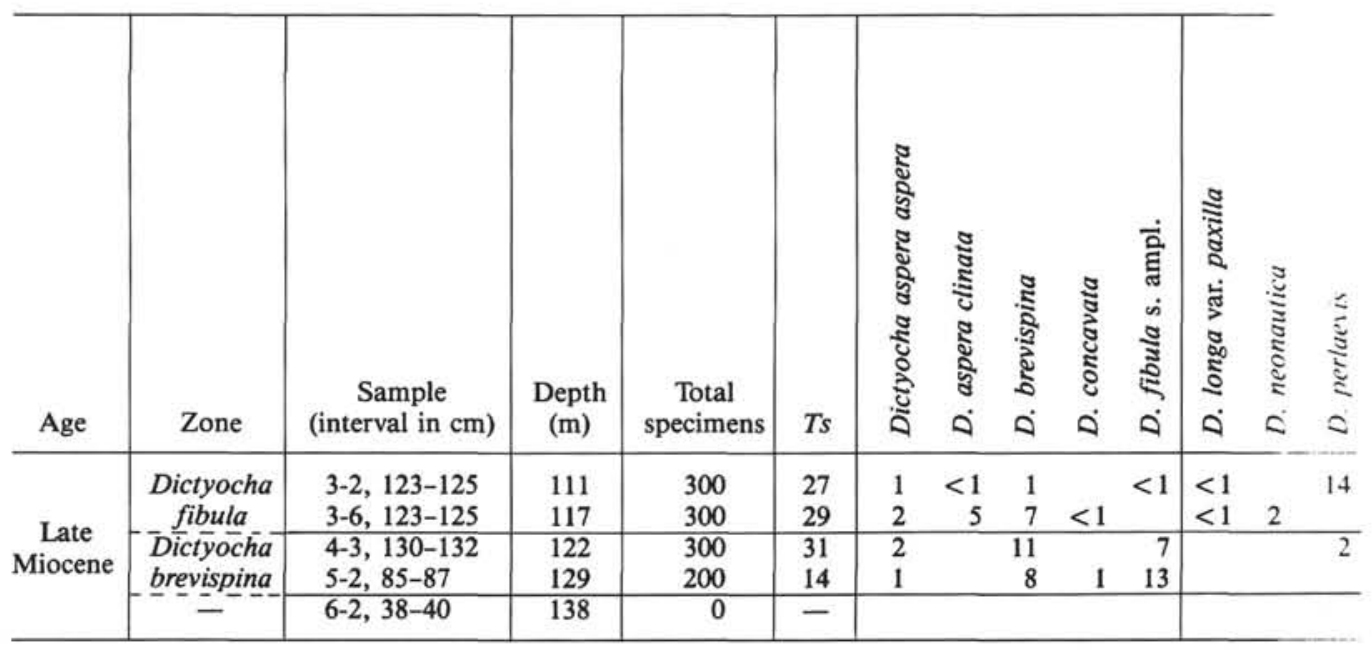

graphic changes indicated by parallel trends for silicoflagellate relative paleotemperatures (Bukry, 1981a, in press).

The silicoflagellate Miocene/Pliocene boundary is not well established because of ecologically variable assemblages and limited reference localities. Events for several species near the boundary could be identified in Hole 552A assemblages, including an uppermost Miocene HOS (highest occurrence surface) for Dictyocha neonautica Bukry (Bukry, $1981 b$, in press), a lower lower Pliocene HOS or last common occurrence for Dictyocha pulchella (Bukry, in press), and a lower lower Pliocene LOS (lowest occurrence surface) for Distephanus jimlingii (Bukry) (Barron, 1976, 1980; Bukry, 1979a). The absence of $D$. neonautica Bukry above Sample 552A-24-3, 98-99 cm was selected because of its proximity to the calcareous nannofossil Miocene/Pliocene boundary and restricted natural range in the Pacific.

The interval from Cores $552 \mathrm{~A}-11$ to $16(53 \mathrm{~m}$ to $73 \mathrm{~m})$ above the lower lower Dictyocha pulchella Subzone has some samples with cool relative paleotemperatures; however the two warmer samples with $T s=66$ and 71 have abundant Dictyocha concinna Bukry which did not appear at eastern equatorial Pacific Sites 503 and 504 until late Pliocene. Relatively warmer paleotemperatures, as indicated by $T s$ values and by lower abundances of Distephanus speculum and Mesocena circulus, favor $D$. concinna at high latitude. The younger occurrences in lower latitudes are not as directly related to paleotemperature peaks. The early Pliocene Dictyocha angulata Subzone is not identified at Site 552 because of the absence of the guide species $D$. angulata Bukry. Cool-water Distephanus dominate the uppermost silicoflagellate-bearing samples from Cores 552A-11 and 12 (53 to $58 \mathrm{~m})$.

Site 552 samples which were processed and examined for silicoflagellates, but which are barren, include: Samples $552-6-2,38-40 \mathrm{~cm}$; $552-12-2,78-80 \mathrm{~cm} ; 552-12-6$, $96-98 \mathrm{~cm} ; 552-13-1,108-110 \mathrm{~cm} ; 552-14-2,89-91 \mathrm{~cm}$; $552-16-1,23-25 \mathrm{~cm}$; 552A-8,CC, 21-22 cm; 552-38A-1, $125-126 \mathrm{~cm}$; and $552 \mathrm{~A}-38-3,10-11 \mathrm{~cm}$.

\section{SITE 553}

\section{$\left(56^{\circ} 05.32\right.$ ' N, $23^{\circ} 20.61^{\prime} \mathrm{W}$, depth $\left.2338-2339 \mathrm{~m}\right)$}

Site 553 is just oceanward of Site 552 , southwest of Hatton-Edoras Bank. No silicoflagellates occur in the Rocella-rich samples available from Cores 553A-8 and 9 (222 to $235 \mathrm{~m}$ ). Sparse to common Eocene silicoflagellates occur in Cores 553A-9 to 11 (235 to $252 \mathrm{~m}$ ). Two samples from Core 11 are assigned to the early Eocene Naviculopsis constricta Zone on the basis of the joint occurrence of Dictyocha deflandrei deflandrei s. ampl., Naviculopsis constricta (Schulz), and N. minor (Schulz), without the younger species in Cores 553A-9 and 10 . Also, one specimen of $D$. deflandrei lobata Bukry, which typified the early to middle Eocene assemblages at North Atlantic Site 385 (Bukry, 1978c) was recorded from Sample 553A-11-5, 7-8 cm, after the counts were completed. This supports a maximum age of early Eocene for Core 553A-11. The presence of Naviculopsis foliacea Deflandre, Mesocena oamaruensis Schulz, and M. occidentalis Hanna ex Bukry in the assemblage from Cores 553A-9 and 10 indicates the younger middle Eocene part of the Naviculopsis foliacea Zone. A larger proportion of the silicoflagellates from Cores 553A-9 to 11 belong to Naviculopsis than to Corbisema, which is thought to reflect relatively cool conditions in Eocene assemblages (Barron et al., in press). Although the species composition of the assemblages changes between cores, the generic ratio of Corbisema to Naviculopsis remains below one (Table 4).

\section{SITE 554}

\section{$\left(56^{\circ} 17.41^{\prime} \mathrm{N}, 23^{\circ} 31.69^{\prime} \mathrm{W}\right.$, depth $\left.2584 \mathrm{~m}\right)$}

Site 554 is on the outer high, oceanward of Sites 552 and 553, that separates Hatton-Edoras Bank structures from normal oceanic crust to the west. Silicoflagellates were recovered in two of the seven samples examined from this site (Table 5). Both belong to the upper Mio- 
Table 2. (Continued).

\begin{tabular}{|c|c|c|c|c|c|c|c|c|c|c|c|c|c|c|c|c|c|c|c|c|c|}
\hline 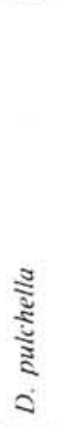 & $\begin{array}{l}\frac{\pi}{3} \\
0 \\
0\end{array}$ & 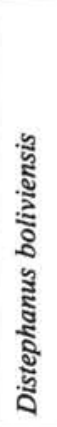 & 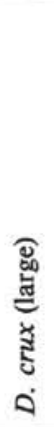 & 造 & 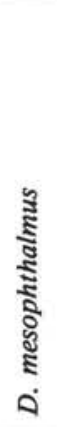 & 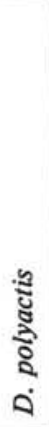 & 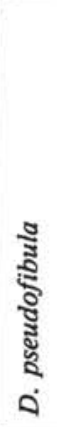 & 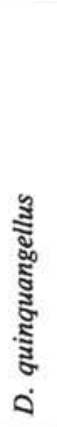 & 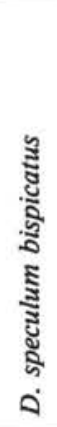 & 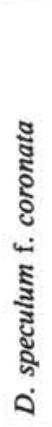 & 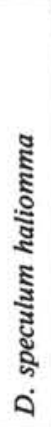 & 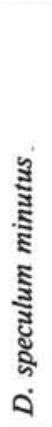 & 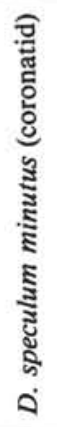 & 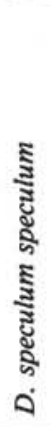 & 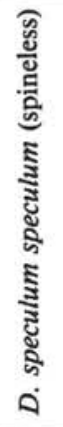 & 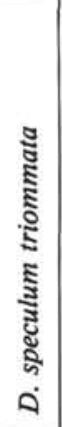 & 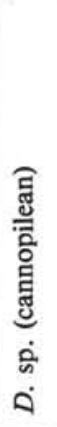 & $\begin{array}{l}\text { है } \\
\text { है } \\
\dot{0}\end{array}$ & 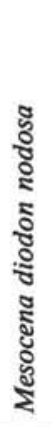 & 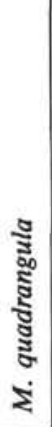 & 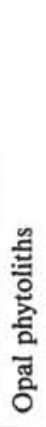 \\
\hline $\begin{array}{l}10 \\
11\end{array}$ & & 3 & 1 & 1 & & & $<1$ & $\begin{array}{l}2 \\
1\end{array}$ & $\begin{array}{r}<1 \\
3\end{array}$ & $\begin{array}{l}3 \\
4\end{array}$ & & $\begin{array}{l}20 \\
27\end{array}$ & $\begin{array}{r}17 \\
3\end{array}$ & $\begin{array}{l}22 \\
26\end{array}$ & $<1$ & $\begin{array}{r}1 \\
<1 \\
\end{array}$ & $\begin{array}{l}2 \\
7\end{array}$ & $\begin{array}{r}3 \\
<1 \\
\end{array}$ & & & $\mathrm{X}$ \\
\hline$<1$ & 1 & $\begin{array}{r}<1 \\
2 \\
\end{array}$ & $\begin{array}{l}1 \\
1 \\
\end{array}$ & & $<1$ & 1 & & & 1 & $\begin{array}{l}1 \\
4 \\
\end{array}$ & & $\begin{array}{r}16 \\
2 \\
\end{array}$ & $\begin{array}{l}2 \\
1 \\
\end{array}$ & $\begin{array}{l}1 \\
58 \\
\end{array}$ & & & $<1$ & $\begin{array}{l}3 \\
1 \\
\end{array}$ & $\begin{array}{r}6 \\
22 \\
\end{array}$ & & \\
\hline
\end{tabular}

Table 3. Miocene and Pliocene silicoflagellate biostratigraphy and relative paleotemperature values $(T S)$ for Cores 11 to 31 of Hole 552A. Abundances shown as percents. Taxa recorded after the counts noted by $A$. Occurrences of taxa in assemblages too sparse for useful percents are noted by $X$, with the dominant taxon circled.

\begin{tabular}{|c|c|c|c|c|c|c|c|c|c|c|c|c|c|c|c|c|c|c|}
\hline \multirow{4}{*}{$\begin{array}{l}\text { Age } \\
\text { Zone } \\
\text { Subzone } \\
\text { Depth }(\mathrm{m})\end{array}$} & \multicolumn{10}{|c|}{ Early Pliocene } & \multicolumn{8}{|c|}{ Late Miocene } \\
\hline & \multicolumn{15}{|c|}{ Dictyocha fibula } & \multirow{2}{*}{\multicolumn{3}{|c|}{$\begin{array}{l}\text { D. fibula or } \\
\text { D. brevispine } \\
-\end{array}$}} \\
\hline & \multicolumn{6}{|c|}{-} & \multicolumn{4}{|c|}{ D. pulchella } & \multicolumn{5}{|c|}{ D. neonautica } & & & \\
\hline & 53 & 58 & 66 & 71 & 73 & 83 & 94 & 98 & 102 & 108 & 112 & 116 & 123 & 128 & 131 & 137 & 140 & 14 \\
\hline (interval in cm) & $\begin{array}{l}\frac{0}{\frac{9}{1}} \\
\frac{0}{2} \\
\stackrel{0}{2} \\
\stackrel{2}{=}\end{array}$ & 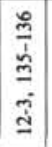 & 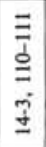 & 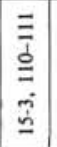 & $\begin{array}{l}\frac{\tilde{m}}{b} \\
\frac{\bar{b}}{6}\end{array}$ & 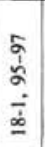 & 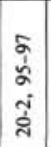 & \begin{tabular}{|l|}
$\frac{\pi}{2}$ \\
$\frac{d}{\frac{1}{2}}$ \\
$\frac{8}{4}$ \\
\end{tabular} & $\begin{array}{l}\frac{8}{1} \\
\frac{8}{3} \\
\text { ป }\end{array}$ & $\begin{array}{l}2 \\
\dot{\alpha} \\
\alpha \\
\dot{j} \\
\dot{n}\end{array}$ & $\begin{array}{l}2 \\
\dot{1} \\
\infty \\
\vdots \\
\frac{1}{2}\end{array}$ & 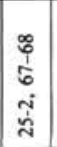 & $\begin{array}{l}\tilde{a} \\
\dot{\alpha} \\
\dot{a} \\
\dot{\alpha}\end{array}$ & 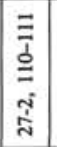 & 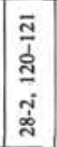 & $\begin{array}{l}\overrightarrow{0} \\
\vdots \\
0 \\
\vdots \\
\vdots \\
\end{array}$ & 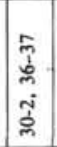 & $\frac{\substack{\frac{m}{1} \\
\frac{m}{m} \\
\frac{i}{m}}}{m}$ \\
\hline $\begin{array}{l}\text { Dictyocha aspera aspera } \\
\text { D. aspera clinata s. ampl. } \\
D \text {. brevispina } \\
D \text {. sp. cf. } D \text {. calida ampliata } \\
D . \text { concinna }\end{array}$ & 1 & $\begin{array}{r}2 \\
<1\end{array}$ & $\begin{array}{r}11 \\
3 \\
50\end{array}$ & $\begin{array}{r}2 \\
10\end{array}$ & $\begin{array}{r}<1 \\
4 \\
36\end{array}$ & $\begin{array}{r}3 \\
13 \\
1 \\
\end{array}$ & 1 & $<1$ & & $\begin{array}{r}1 \\
40\end{array}$ & 7 & 4 & 5 & $\begin{array}{l}2 \\
2\end{array}$ & $\begin{array}{l}2 \\
7 \\
2\end{array}$ & 14 & $\begin{array}{l}10 \\
13\end{array}$ & $\begin{array}{l}x \\
\otimes\end{array}$ \\
\hline $\begin{array}{l}\text { D. constricta } \\
\text { D. fibula s. ampl. } \\
\text { D. longa var. paxilla } \\
\text { D. neonauntica } \\
\text { D. sp. aff. } D \text {. perfecta }\end{array}$ & & 3 & & 12 & 24 & 13 & $\begin{array}{l}<1 \\
<1 \\
<1\end{array}$ & $\begin{array}{l}1 \\
1\end{array}$ & 2 & 2 & 2 & $\begin{array}{l}1 \\
1\end{array}$ & 2 & $\begin{array}{r}<1 \\
1\end{array}$ & 1 & & 5 & 5 \\
\hline $\begin{array}{l}\text { D. perlaevis flexatella } \\
\text { D. perlaevis perlaevis } \\
\text { D. pulchella } \\
\text { D. sp. aff. D. pulchella (angular) } \\
D . \text { sp. cf. D. stapedia aspinosa }\end{array}$ & $\begin{array}{r}<1 \\
1\end{array}$ & $<1$ & 3 & 3 & $<1$ & 1 & $\begin{array}{l}11 \\
14\end{array}$ & ${ }_{21}^{6}$ & $\begin{array}{r}12 \\
17 \\
2\end{array}$ & $\begin{array}{r}1 \\
11 \\
2\end{array}$ & 50 & 39 & 43 & 6 & 1 & $\begin{array}{l}2 \\
2\end{array}$ & & \\
\hline $\begin{array}{l}\text { D. sp. cf. D. stapedia stapedia } \\
\text { D. varia s. ampl. } \\
\text { D. spp. } \\
\text { Distephamus boliviensis } \\
\text { D. crux bispinosus }\end{array}$ & $<1$ & 3 & & $<1$ & $\begin{array}{r}1 \\
3 \\
<1\end{array}$ & 2 & $\begin{array}{l}1 \\
3\end{array}$ & 1 & 24 & $\begin{array}{r}<1 \\
2 \\
1\end{array}$ & 2 & 1 & $\begin{array}{r}<1 \\
1 \\
1\end{array}$ & $<1$ & 2 & $\begin{array}{l}1 \\
1\end{array}$ & 3 & 3 \\
\hline $\begin{array}{l}\text { D. crux crux } \\
\text { D. frugalis } \\
\text { D. jimlingii } \\
\text { D. polyactis } \\
\text { D. pseudofibula } \\
\end{array}$ & $\begin{array}{l}1 \\
3\end{array}$ & $\begin{array}{r}<1 \\
6 \\
10\end{array}$ & 7 & $\begin{array}{l}1 \\
5\end{array}$ & 2 & $\begin{array}{r}3 \\
24 \\
3\end{array}$ & 5 & 1 & 3 & & $\begin{array}{l}1 \\
1\end{array}$ & A & $\begin{array}{l}3 \\
2\end{array}$ & & $\begin{array}{l}1 \\
2\end{array}$ & 4 & 3 & $\mathrm{x}$ \\
\hline $\begin{array}{l}\text { D. quinquangellus } \\
\text { D. speculum bispicatus } \\
\text { D. speculum f. coronata } \\
\text { D. speculum minutus } \\
\text { D. speculum minutus (coronatid) }\end{array}$ & $\begin{array}{r}1 \\
1 \\
<1 \\
1\end{array}$ & $\begin{array}{r}3 \\
1 \\
15 \\
<1 \\
2\end{array}$ & II & $\begin{array}{r}2 \\
25 \\
<1 \\
9\end{array}$ & $\begin{array}{l}6 \\
1 \\
2\end{array}$ & $\begin{array}{r}1 \\
2 \\
10\end{array}$ & $\begin{array}{r}3 \\
6 \\
21\end{array}$ & $\begin{array}{r}3 \\
8 \\
12 \\
3\end{array}$ & $\begin{array}{r}4 \\
2 \\
14 \\
5\end{array}$ & $\begin{array}{r}<1 \\
1 \\
10 \\
1 \\
1\end{array}$ & $\begin{array}{r}1 \\
1 \\
16 \\
2\end{array}$ & $\begin{array}{r}1 \\
8 \\
2 \\
17\end{array}$ & $\begin{array}{r}1 \\
1 \\
18\end{array}$ & $\begin{array}{r}<1 \\
6 \\
40 \\
1\end{array}$ & $\begin{array}{r}1 \\
4 \\
13 \\
13\end{array}$ & $\begin{array}{l}8 \\
4\end{array}$ & $\begin{array}{l}4 \\
5\end{array}$ & $\mathrm{x}$ \\
\hline $\begin{array}{l}\text { D. speculum speculum } \\
\text { D. speculum speculum (spineless) } \\
\text { D. speculum triommata } \\
\text { D. sulcatus } \\
D \text {. sp. (cannopilean) }\end{array}$ & $\begin{array}{r}75 \\
1 \\
<1\end{array}$ & $\begin{array}{r}43 \\
\\
< \\
<1\end{array}$ & 10 & $\begin{array}{r}21 \\
2 \\
3\end{array}$ & $\begin{array}{r}18 \\
1 \\
<1\end{array}$ & 19 & $\begin{array}{r}32 \\
1 \\
2\end{array}$ & 28 & 7 & 24 & 18 & 24 & $\begin{array}{r}16 \\
2 \\
1\end{array}$ & $\begin{array}{r}29 \\
1 \\
12\end{array}$ & $\begin{array}{r}41 \\
1 \\
4\end{array}$ & $\begin{array}{r}56 \\
4 \\
2\end{array}$ & 58 & $3 x$ \\
\hline $\begin{array}{l}\text { D. xenus } \\
\text { Mesocena circulus } \\
\text { M. diodon nodosa } \\
\text { M. quadrangula } \\
\text { M. triangula } \\
\end{array}$ & 4 & 4 & & 4 & & $\begin{array}{r}4 \\
<1 \\
1\end{array}$ & & & 2 & & 2 & & 1 & $<1$ & $\begin{array}{l}1 \\
1\end{array}$ & 2 & 2 & $\begin{array}{l}x \\
x\end{array}$ \\
\hline Total specimens & 300 & 300 & 100 & 300 & 300 & 300 & 300 & 300 & 200 & 300 & 200 & 300 & 300 & 300 & 200 & 100 & 200 & 25 \\
\hline Relative paleotemperature value & 13 & 10 & 71 & 27 & 66 & 32 & 30 & 38 & 63 & 59 & 60 & 45 & 55 & 12 & 16 & 19 & 30 & - \\
\hline
\end{tabular}


Table 4. Eocene silicoflagellate biostratigraphy of Cores 9 to 11 for Hole DSDP 553A. Abundances shown as percents. The Corbisema/Naviculopsis ratio $(\mathrm{C} / \mathrm{N})$ is less than one, suggesting relatively cool conditions in Eocene assemblages. Barren samples examined from Hole 553A included Core-Sections $8, \mathrm{CC}, 8-4,9-1,9-4$, and 9-5.

\begin{tabular}{|c|c|c|c|c|c|c|c|c|c|c|c|c|c|c|c|c|c|c|c|c|c|c|c|c|c|c|c|c|c|}
\hline Age & Zone & $\begin{array}{c}\text { Sample } \\
\text { (interval in } \mathrm{cm} \text { ) }\end{array}$ & $\begin{array}{l}\text { Depth } \\
\text { (m) }\end{array}$ & $\begin{array}{c}\text { Total } \\
\text { specimens }\end{array}$ & $\mathrm{C} / \mathrm{N}$ & 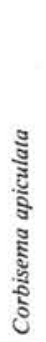 & 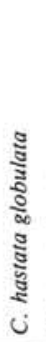 & 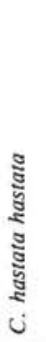 & 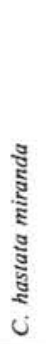 & 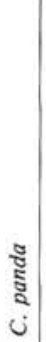 & 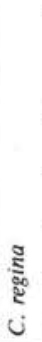 & 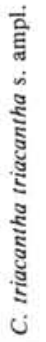 & $\begin{array}{l}\text { iे } \\
\dot{0}\end{array}$ & 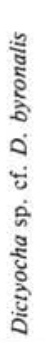 & 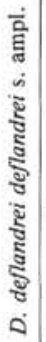 & 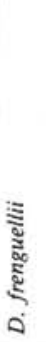 & 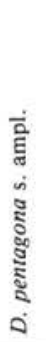 & 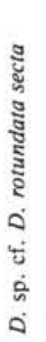 & $\begin{array}{l}0 \\
\text { के } \\
0\end{array}$ & 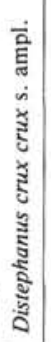 & 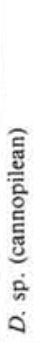 & 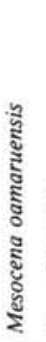 & 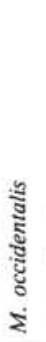 & 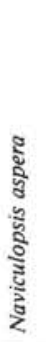 & 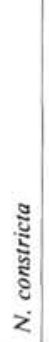 & 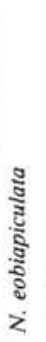 & 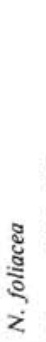 & 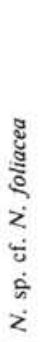 & $\begin{array}{l}\text { ఏ } \\
\text { हे } \\
z\end{array}$ \\
\hline Middle & $N$. & $9-6,56-58$ & 235 & 45 & 0.26 & 7 & & & & & & 4 & & 29 & & & 2 & & 7 & 2 & & 4 & & & 40 & & 2 & & \\
\hline Eocene & foliacea & $\frac{10-1,140-142}{10-5,140-142}$ & $\frac{238}{244}$ & $\begin{array}{r}92 \\
0\end{array}$ & 0.44 & 14 & & & & 2 & 3 & ? & & 17 & & & 4 & & 1 & 2 & & & 5 & & 40 & & 8 & & \\
\hline Early & $\bar{N}$ & $11-4,131-133$ & 252 & 200 & 0.22 & & & 9 & & & & 4 & & 16 & 2 & 2 & 2 & 6 & & & 2 & & & 16 & 25 & 4 & & 1 & 14 \\
\hline Eocene & constricta & $11-5,7-8$ & 252 & 150 & 0.53 & & 1 & 10 & 2 & & & 6 & 5 & 21 & 3 & 5 & 1 & & 1 & & 1 & & & 5 & 23 & 3 & & 2 & 12 \\
\hline
\end{tabular}

Table 5. Late Miocene silicoflagellate biostratigraphy and relative paleotemperature values ( $T S$ ) for DSDP Site 554. Abundances shown as percents. Presence of reworked taxa noted by $R$.

\begin{tabular}{|c|c|c|c|c|c|c|c|}
\hline \multirow{4}{*}{ Age } & \multicolumn{7}{|c|}{ Late } \\
\hline & - & \multicolumn{2}{|c|}{ Miocene } & \multicolumn{4}{|c|}{-} \\
\hline & \multirow[b]{2}{*}{-} & \multirow{2}{*}{\multicolumn{2}{|c|}{$\begin{array}{l}\text { D. brevis- } \\
\text { pina }\end{array}$}} & \multirow{2}{*}{\multicolumn{4}{|c|}{ - }} \\
\hline & & & & & & & \\
\hline Depth $(m)$ & 61 & 71 & 76 & 86 & 96 & 107 & 115 \\
\hline $\begin{array}{r}\text { Sample } \\
\text { (interval in cm) }\end{array}$ & 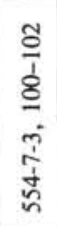 & $\begin{array}{l}\cong \\
\vdots \\
\vdots \\
\vdots \\
j \\
\infty \\
0 \\
\dot{1} \\
n\end{array}$ & 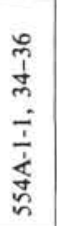 & 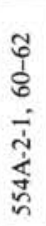 & 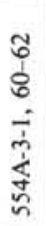 & 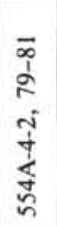 & 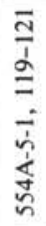 \\
\hline $\begin{array}{l}\text { Cannopilus sp. } \\
\text { Corbisema hastata hastata } \\
\text { Dictyocha aspera aspera } \\
\text { D. brevispina } \\
\text { D. fibula }\end{array}$ & & $\begin{array}{r}4 \\
15 \\
1\end{array}$ & $\begin{array}{l}1 \mathrm{R} \\
19\end{array}$ & & & & \\
\hline $\begin{array}{l}\text { D. pulchella } \\
\text { D. spp. } \\
\text { Distephanus boliviensis } \\
\text { D. crux crux (large) } \\
\text { D. frugalis s. ampl. }\end{array}$ & & $\begin{array}{r}11 \\
1 \\
14\end{array}$ & $\begin{array}{l}1 \\
2 \\
3\end{array}$ & & & & \\
\hline $\begin{array}{l}\text { D. quinquangellus } \\
\text { D. speculum f. coronata } \\
\text { D. speculum minutus } \\
\text { D. speculum minutus (coronatid) } \\
\text { D. speculum speculum }\end{array}$ & & $\begin{array}{r}4 \\
2 \\
4 \\
42 \\
4\end{array}$ & $\begin{array}{r}1 \\
5 \\
27\end{array}$ & & & & \\
\hline $\begin{array}{l}D . \text { sp. cf. } D \text {. stradneri } \\
\text { Mesocena circulus } \\
M \text {. diodon nodosa } \\
M \text {. triodon } \\
\text { Naviculopsis constricta }\end{array}$ & & & $\begin{array}{r}4 \\
5 \\
37 \\
1 \\
1 \mathrm{R}\end{array}$ & & & & \\
\hline Total specimens & 0 & 100 & 202 & 0 & 0 & 0 & 0 \\
\hline Relative paleotemperature value & - & 23 & 22 & - & - & - & - \\
\hline
\end{tabular}

cene Dictyocha brevispina Zone, but cool-water conditions are implied by the high abundances of Distephanus. Sample 554A-1-1, 34-36 cm contains reworked specimens of Paleogene species.

\section{SITE 555}

\section{$\left(56^{\circ} 33.70^{\prime} \mathrm{N}, 2^{\circ} 46.93^{\prime} \mathrm{W}\right.$, depth $\left.1669 \mathrm{~m}\right)$}

Site 555 is between Hatton Bank and Edoras Bank, east of Sites 552 to 554 . The goal of the coring was to compare subsidence history of the banks to the flank sites. Silicoflagellates of Miocene age occur in only 6 of the 17 samples examined (Table 6). The three deepest samples in Cores 18 to 21 (198 to $226 \mathrm{~m}$ ) contain abundant specimens assigned to the middle Miocene Distephanus stauracanthus Subzone, based on the presence of sparse Corbisema triacantha triacantha (Ehrenberg) and Distephanus stauracanthus (Ehrenberg). The upper three samples in Cores 5 to $8(42$ to $71 \mathrm{~m})$ overlie a silicoflagellate-barren interval exceeding $100 \mathrm{~m}$. These samples are dominated by Distephanus speculum speculum (Ehrenberg) and asperoid Dictyocha, indicating assignment to the upper Miocene Dictyocha brevispina Zone. $T s$ values from the 20 s to the 40 s for the $D$. brevispina Zone at Site 555 are similar to those recorded for 552 and 554 , suggesting moderately cool conditions.

\section{SITE 406}

\section{$\left(5^{\circ} 15.50^{\prime} \mathrm{N}, 2^{\circ} 05.41^{\prime} \mathrm{W}, 2907 \mathrm{~m}\right.$ depth)}

Site 406 of Leg 48 was cored just south of the eastwest-trending transform fault which demarcates part of southwest margin of the Rockall Plateau area. Silicoflagellates were studied from 16 samples in Cores 1 to 40 between 0 and $720 \mathrm{~m}$ for possible correlation to the assemblages recovered to the north during Leg 81. Aside from lower Pliocene DSDP Sample 406-4-1, 70-71 cm $(215 \mathrm{~m})$, which correlates very closely to DSDP Sample 552A-18-1, 95-97 cm (83 m), all of the other silicoflagellate assemblages from Site 406 were not recognized from Leg 81 cores. These include the unzoned lower Miocene from Cores 25 to 30, upper Oligocene Naviculopsis biapiculata Zone from Cores 31 to 33 , and the upper Eocene Dictyocha hexacantha Zone from Cores 39 to 40 (Table 7). 
Table 6. Miocene silicoflagellate biostratigraphy and relative paleotemperature values $(T S)$ for Cores 5 to 22 of DSDP Site 555. Abundances shown as percents.

\begin{tabular}{|c|c|c|c|c|c|c|c|c|c|c|c|c|c|c|c|c|c|}
\hline Age & \multicolumn{4}{|c|}{ Late Miocene } & \multicolumn{8}{|c|}{ - } & \multicolumn{5}{|c|}{ Middle Miocene } \\
\hline Zone & \multicolumn{4}{|c|}{ D. brevispina } & \multicolumn{8}{|c|}{-} & \multicolumn{5}{|c|}{ C. triacantha } \\
\hline Subzone & \multicolumn{12}{|c|}{-} & \multicolumn{5}{|c|}{ D. stauracanthus } \\
\hline Depth (m) & 42 & 50 & 59 & 71 & 92 & 109 & 133 & 140 & 158 & 168 & 183 & 189 & 198 & 207 & 215 & 226 & 235 \\
\hline Taxa $\quad \begin{array}{r}\text { Sample } \\
\text { (interval in } \mathrm{cm} \text { ) }\end{array}$ & $\begin{array}{l}\vec{z} \\
\stackrel{1}{2} \\
\dot{0} \\
\dot{n}\end{array}$ & 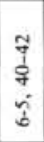 & 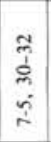 & $\begin{array}{l}\tilde{0} \\
0 \\
8 \\
6 \\
\infty\end{array}$ & $\frac{\frac{\pi}{\partial}}{\frac{1}{2}}$ & $\begin{array}{l}\infty \\
0 \\
0 \\
\infty \\
0 \\
0 \\
0\end{array}$ & $\begin{array}{l}\frac{n}{i} \\
\frac{i}{2} \\
\dot{m} \\
\vdots\end{array}$ & 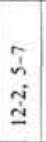 & 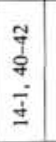 & $\begin{array}{c}a \\
\dot{1} \\
\frac{1}{2}\end{array}$ & 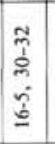 & 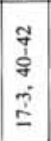 & $\begin{array}{l}\stackrel{n}{i} \\
\tilde{F} \\
\tilde{n} \\
\stackrel{\infty}{\infty}\end{array}$ & $\begin{array}{l}1 \\
\infty \\
\infty \\
\infty \\
N \\
\alpha \\
\alpha\end{array}$ & $\begin{array}{l}\infty \\
\infty \\
1 \\
\infty \\
\dot{j} \\
\tilde{d}\end{array}$ & 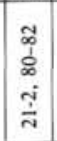 & $\begin{array}{l}\text { สี } \\
\text { ภิ } \\
\text { สี่ }\end{array}$ \\
\hline $\begin{array}{l}\text { Cannopilus } \mathrm{sp} . \\
\text { Corbisema triacantha triacantha } \\
\text { Dictyocha aspera aspera } \\
\text { D. sp. cf. D. aspera clinata } \\
\text { D. brevispina }\end{array}$ & $\begin{array}{l}30 \\
11 \\
\end{array}$ & & $\begin{array}{r}2 \\
19 \\
\end{array}$ & $\begin{array}{r}1 \\
1 \\
11 \\
\end{array}$ & & & & & & & & & $\begin{array}{l}1 \\
2\end{array}$ & & & $\begin{array}{l}2 \\
1\end{array}$ & \\
\hline $\begin{array}{l}\text { D. brevispina (naviculopsid) } \\
\text { D. brevispina (small-portal giant) } \\
\text { D. fibula } \\
\text { D. pulchella } \\
\text { D. pulchella var. inflata }\end{array}$ & & & & $\begin{array}{r}12 \\
4\end{array}$ & & & & & & & & & $\begin{array}{r}1 \\
41 \\
5\end{array}$ & $\begin{array}{r}<1 \\
9 \\
1\end{array}$ & & $\begin{array}{r}10 \\
1 \\
\end{array}$ & \\
\hline $\begin{array}{l}\text { D. spp. } \\
\text { Distephanus boliviensis } \\
\text { D. crux crux s, ampl. } \\
\text { D. crux parvus } \\
\text { D. crux scutulatus }\end{array}$ & $\begin{array}{l}1 \\
5\end{array}$ & & 1 & $\begin{array}{l}1 \\
2\end{array}$ & & & & & & & & & $\begin{array}{r}<1 \\
17 \\
2\end{array}$ & $\begin{array}{r}32 \\
1 \\
<1\end{array}$ & & $\begin{array}{l}1 \\
2 \\
1\end{array}$ & \\
\hline $\begin{array}{l}\text { D. sp. aff. D. frugalis (small) } \\
\text { D. longispinus } \\
\text { D. polyactis } \\
D . \text { quinquangellus } \\
\text { D. speculum bispicatus }\end{array}$ & 2 & & $\begin{array}{l}1 \\
1\end{array}$ & $\begin{array}{r}10 \\
1\end{array}$ & & & & & & & & & $\begin{array}{r}1 \\
14\end{array}$ & 2 & & 10 & \\
\hline $\begin{array}{l}\text { D. speculum f. coronata } \\
\text { D. speculum diommata } \\
\text { D. speculum giganteus } \mathrm{s} \text {. ampl. } \\
\text { D. speculum haliomma } \\
\text { D. speculum minutus }\end{array}$ & 2 & & 7 & 11 & & & & & & & & & & $\begin{array}{r}<1 \\
1 \\
<1\end{array}$ & & & \\
\hline $\begin{array}{l}\text { D. speculum minutus (coronatid) } \\
\text { D. speculum patulus } \\
\text { D. speculum speculum } \\
\text { D. speculum speculum (short spines) } \\
\text { D. speculum triommata }\end{array}$ & $\begin{array}{c}7 \\
18 \\
1\end{array}$ & & $\begin{array}{r}49 \\
1\end{array}$ & $\begin{array}{r}5 \\
39 \\
2\end{array}$ & & & & & & & & & $\begin{array}{r}<1 \\
3\end{array}$ & $\begin{array}{r}4 \\
24\end{array}$ & & $\begin{array}{l}6 \\
1\end{array}$ & \\
\hline $\begin{array}{l}\text { D. stauracanthus } \\
\text { D. stauracanthus (bar) } \\
\text { D. staurodon } \\
\text { D. stradneri } \\
\text { D. xenus }\end{array}$ & 11 & & 3 & 1 & & & & & & & & & & $\begin{array}{r}<1 \\
2\end{array}$ & & $\begin{array}{r}3 \\
40 \\
2\end{array}$ & \\
\hline $\begin{array}{l}\text { Macrora stella } \\
\text { Mesocena apiculata curvata } \\
\text { M. apiculata glabra } \\
\text { M. circulus } \\
M \text {. sp. cf. } M \text {. diodon nodosa }\end{array}$ & & & & 2 & & & & & & & & & 1 & $\begin{array}{l}2 \\
1\end{array}$ & & $\begin{array}{r}<1 \\
2 \\
1 \\
16 \\
1\end{array}$ & \\
\hline $\begin{array}{l}\text { M. quadrangula } \\
\text { M. triangula } \\
\text { M. triodon }\end{array}$ & 8 & & & & & & & & & & & & $\begin{array}{r}1 \\
<1 \\
\end{array}$ & & & & \\
\hline Total specimens & 200 & 0 & 100 & 200 & 0 & 0 & 0 & 0 & 0 & 0 & 0 & 0 & 300 & 300 & 0 & 300 & 0 \\
\hline Relative paleotemperature value & 42 & - & 21 & 34 & - & - & - & - & - & - & - & - & 64 & 50 & - & 38 & - \\
\hline
\end{tabular}

The lower Pliocene silicoflagellate assemblage of Sample 406-4-1, 70-71 cm has most species in common with Sample 552A-18-1, 95-97 cm, at similar abundances (see Table 3). The local ranges of certain ecologically sensitive species, such as Distephanus polyactis, Mesocena circulus, and M. quadrangula, help to key the correlation. A stratigraphic position in the lower Pliocene, somewhat above the Pliocene/Miocene boundary is indicated by the occurrence of Distephanus jimlingii and Dictyocha concinna without D. pulchella. A further similarity is the slightly greater abundance of asperoid over fibuloid Dictyocha. The Dictyocha/Distephanus ratios of 0.9 for Hole 406 and 0.5 for Hole 552A could suggest warmer conditions at Hole 406. But the calculated relative paleotemperature values (Bukry, 1981a and in press) are $T s=46$ for Hole 406 and $T s=32$ for Hole 552A, which can represent within-zone fluctuation. Based on a count of 200 specimens, the assem- blage of Sample 406-4-1, 70-71 cm contains: Dictyocha brevispina $21 \%, D$. concinna $15 \%, D$. longa $2 \%, D$. longa var. paxilla $2 \%, D$. perlaevis perlaevis $1 \%, D$. varia $1 \%, D$. sp. (fibuloid) $2 \%$, Distephanus boliviensis $1 \%$, D. frugalis $5 \%, D$. frugalis (triommatid) $5 \%, D$. frugalis (cannopilean) $3 \%, D$. jimlingii $6 \%, D$. polyactis $1 \%, D$. quinquangellus $2 \%, D$. speculum $\mathrm{f}$. coronata $4 \% D$. speculum speculum $18 \%, D$. sp. (cannopilean) $3 \%$, D. sp. (cruxoid) $1 \%$, Mesocena circulus $10 \%$, and M. quadrangula $2 \%$.

Upper Eocene Dictyocha hexacantha Zone assemblages in Cores 39 and 40 of Hole 406 from the Rockall Plateau area have common Corbisema apiculata, Dictyocha hexacantha, Mesocena apiculata apiculata, and Naviculopsis constricta s. ampl. The Corbisema/Naviculopsis ratio $(\mathrm{C} / \mathrm{N})$, which appears to indicate warmer paleotemperatures for values greater than one (Barron et al., in press), is $\mathrm{C} / \mathrm{N}=0.83$ for Core 39 and $\mathrm{C} / \mathrm{N}=1.0$ 
Table 7. Eocene to Miocene silicoflagellate biostratigraphy for Cores 25 to 40 of DSDP Hole 406. Abundances shown as percents. Occurrences of taxa in assemblages too sparse for useful percents are noted by $\mathrm{X}$, with dominant taxa circled. (Occurrence of diatom Rocella gelida is shown: ${ }^{*}=$ present, $\mathrm{A}=$ acme.)

\begin{tabular}{|c|c|c|c|c|c|c|c|c|c|c|c|}
\hline Age & \multicolumn{5}{|c|}{ Early Miocene? } & \multirow{2}{*}{\multicolumn{3}{|c|}{$\begin{array}{l}\text { Late Oligocene } \\
\text { N. biapiculata }\end{array}$}} & \multicolumn{3}{|c|}{ Late Eocene } \\
\hline Zone & \multicolumn{5}{|c|}{-} & & & & $\xi-$ & $\begin{array}{l}\text { D. } h \\
\text { can }\end{array}$ & $\begin{array}{l}\text { hexa } \\
\text { ntha }\end{array}$ \\
\hline Subzone & & & - & & & \multicolumn{3}{|c|}{$\begin{array}{c}\text { D. speculum } \\
\text { haliomma }\end{array}$} & $\{-$ & \multicolumn{2}{|c|}{ - } \\
\hline Depth (m) & 566 & 585 & 594 & 604 & 615 & 624 & 635 & 643 & 695 & 705 & 710 \\
\hline Taxa & 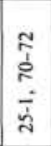 & 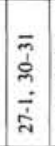 & 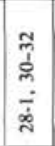 & कृ & 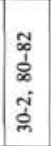 & $\begin{array}{l}\frac{8}{1} \\
\frac{1}{0} \\
\frac{1}{m}\end{array}$ & 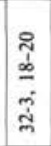 & $\begin{array}{l}\bar{a} \\
\dot{\alpha} \\
\bar{j} \\
\bar{m}\end{array}$ & $\begin{array}{l}\equiv \\
\vdots \\
0 \\
\vdots \\
\dot{0} \\
\infty\end{array}$ & 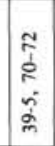 & 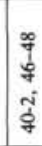 \\
\hline $\begin{array}{l}\text { Corbisema apiculata } \\
\text { C. archangelskiana } \\
\text { C. flexuosa } \\
\text { C. hastata hastata } \\
\text { C. hastata miranda }\end{array}$ & \multicolumn{5}{|l|}{$\otimes$} & \multicolumn{3}{|c|}{1} & $\mathrm{x}$ & $\begin{array}{l}12 \\
3 \\
1\end{array}$ & $\begin{array}{r}17 \\
2 \\
2\end{array}$ \\
\hline $\begin{array}{l}\text { C. katharinae } \\
\text { C. recta } \\
\text { C. regina } \\
\text { C. triacantha mediana } \\
\text { C. triacantha triacantha }\end{array}$ & & $\mathrm{x}$ & $\mathrm{x}$ & 6 & 15 & \multirow{2}{*}{\multicolumn{3}{|c|}{$\mathrm{x}$}} & $\mathrm{x}$ & $\begin{array}{l}1 \\
2\end{array}$ & 2 \\
\hline $\begin{array}{l}\text { Dictyocha sp. cf. D. brevispina } \\
D \text {. sp. aff. D. byronalis } \\
D \text {. deflandrei deflandrei } \\
D \text {. hexacantha } \\
D . \text { sp. }\end{array}$ & & & & & & & & & $\mathrm{x}$ & $\begin{array}{r}1 \\
31 \\
1 \\
\end{array}$ & $\begin{array}{l}17 \\
7 \\
6\end{array}$ \\
\hline $\begin{array}{l}\text { Distephanus crux crux s. ampl. } \\
\text { D. crux darwinii } \\
\text { D. hannai s. ampl. } \\
\text { D. speculum haliomma } \\
\text { D. speculum hemisphaericus }\end{array}$ & $\mathrm{x}$ & $\begin{array}{l}x \\
x \\
x\end{array}$ & $\begin{array}{l}x \\
x\end{array}$ & $\begin{array}{r}26 \\
1\end{array}$ & 9 & $\begin{array}{l}\otimes \\
x\end{array}$ & $\begin{array}{r}30 \\
6 \\
1 \\
8 \\
2\end{array}$ & $\begin{array}{l}9 \\
1 \\
3\end{array}$ & & & \\
\hline $\begin{array}{l}\text { D. speculum patulus } \\
\text { D. sp. (cannopilean, large) } \\
\text { Lyramula? sp. } \\
\text { Macrora stella } \\
\text { Mesocena apiculata apiculata }\end{array}$ & $\begin{array}{l}x \\
\otimes\end{array}$ & $\mathrm{x}$ & $\mathrm{x}$ & $\begin{array}{r}1 \\
4 \\
4 \\
4\end{array}$ & $\begin{array}{r}2 \\
2 \\
1 \\
35\end{array}$ & $\begin{array}{l}x \\
x\end{array}$ & $\begin{array}{l}2 \\
2 \\
2 \\
3\end{array}$ & $\begin{array}{l}2 \\
1 \\
8\end{array}$ & & 24 & 14 \\
\hline $\begin{array}{l}\text { M. apiculata apiculata (spineless) } \\
\text { M. apiculata curvata } \\
\text { M. apiculata evexa } \\
\text { M. apiculata glabra } \\
\text { M. oamaruensis? }\end{array}$ & $x$ & $\mathrm{x}$ & $\otimes$ & $\begin{array}{l}2 \\
9\end{array}$ & $\begin{array}{l}3 \\
4\end{array}$ & $x$ & 2 & $\begin{array}{l}2 \\
1\end{array}$ & $\mathrm{x}$ & & \\
\hline $\begin{array}{l}\text { M. occidentalis } \\
\text { M. sp. } \\
\text { Naviculopsis biapiculata } \\
\text { N. constricta s. ampl. } \\
\text { N. eobiapiculata } \\
\end{array}$ & $\mathrm{x}$ & $\begin{array}{l}\otimes \\
\mathrm{x} \\
\mathrm{x}\end{array}$ & $\begin{array}{l}x \\
x\end{array}$ & $\begin{array}{r}20 \\
6\end{array}$ & 12 & $\begin{array}{l}x \\
x\end{array}$ & $\begin{array}{r}29 \\
3\end{array}$ & $\begin{array}{l}34 \\
40 \\
\end{array}$ & $\otimes$ & $\begin{array}{r}2 \\
21 \\
2 \\
\end{array}$ & 18 \\
\hline $\begin{array}{l}\text { N. foliacea } \\
N \text {. sp. cf. } N \text {, lata (narrow) } \\
N \text {. pacifica pansa } \\
N \text {. sp. } \\
\end{array}$ & $\mathrm{x}$ & & & $\begin{array}{l}8 \\
1\end{array}$ & & & & & $\mathrm{x}$ & & 2 \\
\hline Total specimens & 20 & 27 & 22 & 80 & 100 & 19 & 100 & 175 & 26 & 200 & 65 \\
\hline Diatom: Rocella gelida & & & $\cdot$ & & $\cdot$ & & $\cdot$ & & & & \\
\hline
\end{tabular}

for Core 40. This suggests some cooling for upper Eocene assemblages here, when considered with the $\mathrm{C} / \mathrm{N}$ $=0.25$ value calculated for unzoned Core 38 . These values are similar to those from the Kellogg Shale and Kreyenhagen Formation of California, which also contains $D$. hexacantha Zone silicoflagellates. The effectiveness of the $\mathrm{C} / \mathrm{N}$ ratio for middle and upper Eocene paleotemperature trends is still being tested (Barron et al., in press).

The upper Oligocene assemblages of Cores 31 to 33 from Hole 406 are assigned to the Distephanus speculum haliomma Subzone, which was not recovered by Leg 81 coring. A distinctive new subspecies, Mesocena apiculata evexa, first occurs in this interval with the acme of diatom Rocella gelida, just below the Oligocene/ Miocene boundary. The ratio of Rocella/silicoflagellates is 19.0 in the acme Sample 406-33-1, 90-91 cm (643 $\mathrm{m})$. This is a high value relative to a Rocella/silicoflagel- late ratio of 18.7 for the much more strongly dissolved coeval assemblage from western North Atlantic Site 385 , because Rocella is very resistant to dissolution. The presence of reworked Lyramula, a Cretaceous silicoflagellate, in this sample duplicates the occurrence in DSDP Site 385, Cores 2 and 3, in the western North Atlantic (Bukry, 1978c).

Silicoflagellates in the unzoned lower Miocene interval of Cores 25 to 30 from Hole 406 are probably quite low in the lower Miocene because they are just above the diatom Rocella acme and contain an abundance of older taxa, such as Mesocena apiculata apiculata and Naviculopsis biapiculata. In addition, $N$. pacifica pansa, having flared cylindrical spines, is common in Core 30. This species has previously been illustrated from the North Atlantic in DSDP Sample 369A-13-5, 71-72 cm $(163 \mathrm{~m})$, which was correlated at the Oligocene/Miocene boundary in the lower Triquetrorhabdulus carinatus Zone of coccoliths (Bukry, 1978a). A new taxon, Mesocena apiculata evexa, occurs through Cores 25 to 30 at DSDP 406, and with the presence of Naviculopsis sp. cf. $N$. lata (narrow), probably an early form derived from $N$. biapiculata, shows a close correlation to DSDP Sample 403-26-2, $50-52 \mathrm{~cm}(235 \mathrm{~m})\left(56^{\circ} 08.31^{\prime} \mathrm{N}, 23^{\circ}\right.$ $17.64^{\prime} \mathrm{W}, 2317 \mathrm{~m}$ ) from the western margin of Rockall Plateau where Site 552 was cored by Leg 81 . An unconformity at $173 \mathrm{~m}$ depth at Site 552 juxtaposes middle Miocene above upper Oligocene, so no continuous biosiliceous Oligocene/Miocene boundary interval is preserved in Leg 81 sites. The lower lower Miocene assemblage of Sample 403-26-2, 50-52 cm includes: Corbisema triacantha mediana $3 \%, C$. triacantha triacantha $10 \%$, Distephanus crux crux s. ampl. $21 \%, D$. crux darwinii $2 \%, D$. speculum hemisphaericus $3 \%$, D. speculum patulus $6 \%, D$. staurodon $4 \%, D$. sp. (large, seven-spined) $3 \%$, Macrora stella 4\%, Mesocena apiculata apiculata $1 \%, M$. apiculata curvata 4\%, M. apiculata evexa $17 \%$, Naviculopsis biapiculata $13 \%, N$. constricta s. ampl. $1 \%, N$. eobiapiculata $2 \%, N$. sp. cf. $N$. lata (narrow) $5 \%$, and $N$. sp. $1 \%$.

\section{CONCLUSIONS}

Neogene silicoflagellate assemblages from the Rockall Plateau region show many species and range relations which have also been recorded from the eastern equatorial Pacific. For instance, the temporal sequence from last Dictyocha neonautica to last common D. pulchella, to first $D$. stapedia aspinosa occurs in both regions. Also, an angular variant of $D$. pulchella only occurs in the lower part of the Dictyocha pulchella Subzone at both Sites 504 and 552. But the upper Miocene and lower Pliocene assemblages of DSDP Sites 552, 554, and 555 contain more cool-water Distephanus, such as Distephanus jimlingii, which is missing at equatorial Pacific Sites 503 and 504.

The additional numbers of Distephanus are evident in the Ts values, which are cooler for coeval zones and subzones at Rockall Plateau. Also, reversals in the relative abundances of species common to both regions is shown by comparing Distephanus speculum bispicatus and $D$. speculum f. coronata between Holes 504 and 
552A. Even though Rockall assemblages are cooler, the major cool and warm trends of lower latitudes appear to be represented in the silicoflagellate assemblages.

The absence of a DSDP siliceous Miocene/Pliocene boundary sequence from the tropical Atlantic hinders silicoflagellate paleotemperature comparisons for this interval. Instead, the eastern equatorial Pacific, with both oxygen-isotope records (Keigwin, 1979) and quantitative silicoflagellate data (Bukry, in press) showing similar trends, provides a comparison for the Rockall Plateau climate, which was much cooler during cold pulses ( $T s$ values less than half as high), but only slightly cooler during warm periods ( $T S$ values approximately two-thirds as high). A postulated gradual emergence of the Central American land bridge from late Miocene to early Pliocene (Kaneps, 1979) did not cause major differences to develop in the silicoflagellate assemblages compared. Of 30 taxa identified from the Dictyocha pulchella Subzone of equatorial Pacific Site DSDP 504, $53 \%$ occur in coeval samples from Hole 552A. The absence of Dictyocha angulata in the Dictyocha fibula Zone in Hole 552A is as likely the result of thermal exclusion as isolation by a land bridge.

As quantitative silicoflagellate data become available from more biogeographically diverse sites, paleoecologic interpretations can be tested and improved. The present array of data and sites, however, permits recognition of consistent differences and similarities between North Atlantic Rockall Plateau assemblages and those of the eastern equatorial Pacific.

\section{SYSTEMATIC PALEONTOLOGY}

\section{Genus CANNOPILUS Haeckel, 1887 Cannopilus sp.}

Remarks: Taxonomically indeterminate specimens of helmetshaped silicoflagellates are included here.

\section{Genus CORBISEMA Hanna, 1928}

Corbisema apiculata (Lemmermann)

Dictyocha triacantha apiculata Lemmermann, 1901, p. 259, pl. 10, figs. $19,20$.

\section{Corbisema archangelskiana (Schulz)}

Dictyocha triacantha archangelskiana Schulz, 1928, p. 250, fig. 33a-c.

\section{Corbisema flexuosa (Stradner)}

Corbisema triacantha var. flexuosa Stradner, 1961, p. 89, figs. 1-8, fig. $1 \mathrm{C}$.

Corbisema flexuosa (Stradner) Perch-Nielsen, 1975, p. 685, pl. 3, fig. 10.

Corbisema hastata globulata Bukry

Corbisema hastata globulata Bukry, 1976a, p. 892, pl. 4, figs. 1-8.

Corbisema hastata hastata (Lemmermann)

Dictyocha triacantha var. hastata Lemmermann, 1901, p. 259, pl. 10, figs. $16,17$.

\section{Corbisema hastata miranda Bukry}

Corbisema hastata minor (Schulz) Bukry, 1975b, p. 854, pl. 1, fig. 10. Corbisema hastata miranda Bukry, in Barron, Bukry, and Poore, in press.
Corbisema katharinae Bukry

Corbisema katharinae Bukry, 1976b, p. 848, pl. 1, figs. 1-6.

Corbisema panda Bukry, n. sp.

(Plate 1, Figs. 1-3)

Corbisema bukry (Jousé) in Dzinoridze, R. N., et al., 1979 (in part), p. 366, pl. 11, fig. 11 (not fig. 10).

Description: Corbisema panda has rounded apices and inter-apex areas. The star-shaped ring is pentagonal with the two-segment apical bar connected to the ring by struts from the inter-apex areas. Projecting structures, such as spines, pikes, or spires, are absent. The apical structure has little or no elevation above the ring.

Remarks: Corbisema panda is distinguished from large, pentagonal Dictyocha challengeri Martini et Müller (see Martini and Müller, 1976) by the absence of spines and pikes, and by nonsulcate inter-apex areas. Possibly, $C$. panda was derived from $C$. inermis inermis, which was also spineless, but possessed pikes. The portals of $C$. archangelskiana are too elongate, but that species showed a propensity to produce nontrigonal variations, thus suggesting the generic assignment for spineless C. panda. The quadrate specimens of Hannaites quadria Mandra (=Dictyocha quadria (Mandra) Martini et Müller) from the Norwegian Sea that lack bulbs, spines, and pikes (see Martini and Müller, 1976) would resemble $C$. panda if they were in a pentagonal mode. But the elongate portals of those specimens suggest closer affinity with the $C$. archangelskiana plexus.

Occurrence: Corbisema panda is sparse in middle Eocene sample DSDP 553A-10-1, 140-142 cm (238 m) which is assigned to the Naviculopsis foliacea Zone. The synonymy specimen of Dzinoridze et al. (1979) is from the middle or upper Eocene DSDP Sample 338-29-3, $35-37 \mathrm{~cm}(278 \mathrm{~m})$ from the Vøring Plateau, west of Norway.

Size: Maximum internal diameter 80 to $100 \mu \mathrm{m}$ (holotype $88 \mu \mathrm{m}$ ).

Holotype: USNM 358905 (Plate 1, Figs. 1, 2).

Isotype: USNM 358906.

Type locality: Eastern North Atlantic Ocean, DSDP Sample 553A$10-1,140-142 \mathrm{~cm}$

\section{Corbisema recta (Schulz)}

Dictyocha triacantha var. recta Schulz, 1928, p. 250, fig. 32a, b.

\section{Corbisema regina Bukry}

Corbisema triacantha var. minor (Schulz), Ling, 1972 (in part), p. 158, pl. 24, figs. 20-23.

Corbisema regina Bukry, in Barron, Bukry, and Poore, in press.

\section{Corbisema triacantha mediana Bukry}

Corbisema triacantha mediana Bukry, 1978a, p. 696, pl. 1, figs. 8-12.

Corbisema triacantha triacantha (Ehrenberg)

Dictyocha triacantha Ehrenberg, 1844a, p. 80.

\section{Genus DICTYOCHA Ehrenberg, 1837} Dictyocha angulata Bukry

Dictyocha angulata Bukry, 1982a, p. 431, pl. 1, figs. 9-12; pl. 2, fig. 1.

\section{Dictyocha aspera aspera (Lemmermann)}

Dictyocha fibula var. aspera Lemmermann, 1901, p. 260, pl. 10, figs. $27,28$.

Dictyocha aspera (Lemmermann) Bukry et Foster, 1973 (in part), p. 826 , pl. 2 , figs. 4,6 .

\section{Dictyocha aspera clinata Bukry}

Dictyocha aspera clinata Bukry, 1975a, p. 695, pl. 1, figs. 1-5.

\section{Dictyocha brevispina (Lemmermann)}

Dictyocha fibula var. brevispina Lemmermann, 1901, p. 260; Ehrenberg, 1854 (in part), pl. 21, fig. 42 b; pl. 22, fig. $42 \mathrm{a}$, b. 
Dictyocha byronalis Bukry

Dictyocha byronalis Bukry, in Barron, Bukry, and Poore, in press.

\section{Dictyocha calida ampliata Bukry}

Dictyocha calida ampliata Bukry, 1979b, p. 982, pl. 2, figs. 1, 2, 9.

\section{Dictyocha concavata Dumitrică}

Dictyocha concavata Dumitrică, 1978, p. 212, pl. 3, figs. 1-4.

Dictyocha concinna Bukry

Dictyocha concinna Bukry, in press, pl. 2, figs. 5-11. Dictyocha constricta (Schulz)

Dictyocha fibula fa. constricta Schulz, 1928, p. 253, fig. 35a, b.

Dictyocha deflandrei deflandrei Frenguelli ex Glezer

Dictyocha deflandrei Frenguelli, 1940 (in part), p. 65, fig. 14a, d. Dictyocha deflandrei deflandrei Frenguelli, Glezer, 1966, p. 262, pl. 12 , figs. 13,16 ; pl. 32 , fig. 4 .

\section{Dictyocha deflandrei lobata Bukry}

Dictyocha deflandrei lobata Bukry, 1978c, p. 785, pl. 1, figs. 11-17.

\section{Dictyocha fibula Ehrenberg}

Dictyocha fibula Ehrenberg, 1839, fide Loeblich et al., 1968, p. 90, pl. 9, figs. 7-12.

\section{Dictyocha frenguellii Deflandre}

Dictyocha frenguellii Deflandre, 1950, p. 194, figs. 188-193.

\section{Dictyocha hexacantha Schulz}

Dictyocha hexacantha Schulz, 1928, p. 255, fig. 43.

Dictyocha hexacantha Schulz, Bukry, 1975b, p. 855, pl. 4, figs. 1, 2.

\section{Dictyocha longa Bukry}

Dictyocha longa Bukry, 1982a, p. 432, pl. 2, figs. 8-13; pl. 3, figs. 1,

\section{Dictyocha longa var. paxilla Bukry}

Dictyocha longa Bukry var. paxilla Bukry, 1982a, p. 432, pl. 3, figs. 3-8.

\section{Dictyocha neonautica Bukry}

Dictyocha navicula Ehrenberg, Bukry et Foster, 1973 (in part), p. 827, pl. 3 , figs. 6,7 .

Dictyocha neonautica Bukry, 1981b, p. 442.

\section{Dictyocha pentagona (Schulz)}

Dictyocha fibula var. pentagona Schulz, 1928, p. 225, fig. 41a, b.

Dictyocha perfecta Bukry

Dictyocha perfecta Bukry, 1982b, p. 315, pl. 3, figs. 5-10.

\section{Dictyocha perlaevis flexatella Bukry}

Dictyocha perlaevis flexatella Bukry, 1979b, p. 984, pl. 3, figs. 1-3. Dictyocha perlaevis flexatella Bukry, 1979a, p. 561, pl. 2, figs. 7-10.

\section{Dictyocha perlaevis perlaevis Frenguelli}

Dictyocha perlaevis Frenguelli, 1951, p. 279, fig. 4b, c.

Dictyocha perlaevis Frenguelli, Bukry, 1979b, p. 984, pl. 3, figs. 6-11.

\section{Dictyocha pulchella Bukry}

Dictyocha pulchella Bukry, 1975a, p. 687, pl. 4, figs. 1-3. Dictyocha pulchella Bukry, Bukry, 1981a, p. 549, pl. 3, figs. 5, 6.
Dictyocha pulchella Bukry var. inflata Bukry, n. var.

(Plate 1, Figs. 4-7)

Description: Dictyocha pulchella var. inflata has a rounded, nonsulcate basal ring with nearly equant minor and major axes and prominent basal pikes. The apical bar is moderate to long and is aligned with the minor axis. Spines are short.

Remarks: Dictyocha pulchella var. inflata is distinguished from $D$. pulchella by the rounded, nonsulcate ring outline and more nearly equant axes. It is distinguished from $D$. varia by longer bar and less sulcate, more equant ring, and from $D$. brevispina ausonia by larger side portals and rounder ring.

Occurrence: Dictyocha pulchella var. inflata occurs in the middle Miocene Distephanus stauracanthus Subzone of Cores 18 to 21 at DSDP Site 555 between Hatton Bank and Edoras Bank. It is a minor variety (1 to $5 \%$ ), numerically dominated by Dictyocha pulchella in these cores.

Size: Maximum internal length 27 to $35 \mu \mathrm{m}$ (holotype $33 \mu \mathrm{m}$ ).

Holotype: USNM 358907 (Plate 1, Figs. 4, 5).

lsotypes: USNM 358908 and 358909.

Type locality: Rockall Plateau, eastern North Atlantic, DSDP Sample 555-18-2, 73-75 cm.

\section{Dictyocha rotundata secta (Glezer) n. comb.}

Dictyocha rotundata var. secta Glezer, 1962 , p. 152, fig. 5a-k. Dictyocha rotundata secta Glezer, Bukry, 1976b, p. 848, pl. 1, figs. 7-10.

\section{Dictyocha stapedia aspinosa Bukry}

Dictyocha stapedia aspinosa Bukry, 1976c, p. 724, pl. 2, figs. 6-9.

\section{Dictyocha stapedia stapedia Haeckel}

Dictyocha stapedia Haeckel, 1887, p. 1561, pl. 101, figs. 10-12.

Dictyocha stapedia stapedia Haeckel, Bukry, 1979b, p. 984.

Dictyocha stapedia stapedia Haeckel, Bukry, 1976c, p. 724, pl. 3, figs. $1-7$.

\section{Dictyocha varia Locker}

Dictyocha varia Locker, 1975, p. 99, text-figs. 1 and 3.

Dictyocha spp.

Remarks: This category includes miscellaneous specimens not assignable to described taxa, usually because of tilting, obstruction, or breakage of the specimens.

\section{Genus DISTEPHANUS Stöhr, 1880 \\ Distephanus boliviensis (Frenguelli)}

Dictyocha boliviensis Frenguelli, 1940, p. 44, fig. 4.

Distephanus boliviensis (Frenguelli) Bukry et Foster, 1973, p. 827, pl. 4 , figs. 1-3.

\section{Distephanus crux bispinosus Dumitrică}

Distephanus crux spp. bispinosus Dumitrică, 1973, p. 850, pl. 6, figs. $3,6,7$.

Distephanus crux bispinosus Dumitrică, Bukry, in press, pl. 7, fig. 2 .

\section{Distephanus crux crux (Ehrenberg)}

Dictyocha crux Ehrenberg, 1840, p. 207; Ehrenberg, 1854, pl. 18, fig. 56; pl. 20 (1), fig. 46; pl. 33 (15), fig. 9; pl. 33 (16), fig. 9; pl. 33 (17), fig. 5.

Distephanus crux (Ehrenberg), Bukry, 1982a, p. 430.

\section{Distephanus crux darwinii Bukry}

Distephanus crux darwinii Bukry, 1976a, p. 895, pl. 7, figs. 4-13.

\section{Distephanus frugalis (Bukry)}

Distephanus boliviensis frugalis Bukry, 1975a, p. 688, pl. 2, figs. 2-7. Distephanus frugalis (Bukry) Bukry, 1979a, p. 561, pl. 3, figs. 5, 6.

\section{Distephanus hannai (Bukry)}

Distephanus crux hannai Bukry, 1975b, p. 855, pl. 4, figs. 4-6. Distephanus hannai (Bukry) Bukry, 1979a, p. 561. 


\section{Distephanus jimlingii (Bukry)}

Distephanus boliviensis jimlingii Bukry, 1975a, p. 688, pl. 1, figs. 6 , 7 ; pl. 2, fig. 1

Distephanus jimlingii (Bukry) Bukry, 1979a, p. 561. pl. 3, figs. 7-12.

\section{Distephanus polyactis (Ehrenberg)}

(Plate 3, Figs. 8, 9)

Dictyocha polyactis Ehrenberg, 1839, p. 129; Ehrenberg, 1854, pl. 22, fig. 50 .

Distephanus polyactis (Ehrenberg) Deflandre, Bukry et Foster, 1973, p. 828 , pl. 5 , figs. 6,7

\section{Distephanus pseudofibula (Schulz)}

Dictyocha speculum f. pseudofibula Schulz, 1928, p. 262, fig. 51a, b. Distephanus pseudofibula (Schulz) Bukry, 1976b, p. 848.

Distephanus quinquangellus Bukry et Foster

Distephamus quinquangellus Bukry et Foster, 1973, p. 828, pl. 5, fig. 4.

\section{Distephanus speculum bispicatus Bukry}

Distephanus speculum (Ehrenberg) Haeckel subsp. bispicatus Bukry, 1982 b, p. 315 , pl. 6 , figs. $2-4$.

Distephanus speculum f. coronata Schulz

Distephanus speculum f. coronata Schulz, 1928, p. 262, fig. 50.

Distephanus speculum diommata (Ehrenberg)

Dictyocha diommata Ehrenberg, 1845, p. 56, 76; Ehrenberg, 1854, pl. 33 (17), fig. 6.

Distephanus speculum diommata (Ehrenberg) Bukry, 1979a, p. 562.

\section{Distephanus speculum giganteus Bukry}

Distephanus speculum giganteus Bukry, 1976b, p. 848, pl. 1, fig. 19; pl. 2, figs. 1, 2 .

Distephanus speculum giganteus Bukry, Bukry, 1981a, p. 550, pl. 4, figs. 1,2 .

\section{Distephanus speculum haliomma (Ehrenberg)}

Dictyocha haliomma Ehrenberg, 1844a, p. 64, 80; ?Ehrenberg, 1854, pl. 21 , fig. 46.

Distephanus speculum haliomma (Ehrenberg) Bukry, 1978a, p. 697, pl. 2, fig. 10 .

\section{Distephanus speculum hemisphaericus (Ehrenberg)}

Dictyocha hemisphaerica Ehrenberg, 1844b, p. 258, 266; Lemmermann, 1901, pl. 11, fig. 21 (fide Loeblich et al., 1968).

Distephanus speculum hemisphaericus (Ehrenberg) Bukry, 1975b, p. 855 , pl. 4 , fig. 8 .

\section{Distephanus speculum minutus (Bachmann) emend. Bukry}

Dictyocha speculum f. minuta Bachmann in Ichikawa et al., 1967, p. 161 , pl. 7, figs. 12-15.

Distephanus speculum minutus (Bachmann) emended, Bukry, 1981a, p. 550 .

\section{Distephanus speculum patulus Bukry}

Distephanus speculum (Ehrenberg) Haeckel subsp. patulus Bukry, 1982a, p. 433 , pl. 5, figs. $7-10$.

\section{Distephanus speculum speculum (Ehrenberg)}

Dictyocha speculum Ehrenberg, 1839, p. 150; Ehrenberg, 1854, pl. 18 fig. 57 ; pl. 19 , fig. 41 ; pl. 21 , fig. 44 ; pl. 22 , fig. 47.

Distephanus speculum (Ehrenberg) Haeckel, 1887, p. 1565.

\section{Distephanus speculum triommata (Ehrenberg)}

Dictyocha triommata Ehrenberg, 1845, p. 56, 76; Ehrenberg, 1854, pl. 33 (15), fig. 11.
Dictyocha triommata Ehrenberg, Locker, 1974, p. 643, pl. 4, fig. 5 (holotype figured).

Distephanus speculum triommata (Ehrenberg) Bukry, 1976a, p. 896.

\section{Distephanus stradneri (Jerković)}

Dictyocha schauinslandii stradneri Jerković, 1965, p. 3, pl. 2, fig. 2; Stradner, 1961, fig. 60

Distephanus stradneri (Jerković) Bukry, 1978a, p. 698.

\section{Distephanus stauracanthus (Ehrenberg)}

Dictyocha stauracanthus Ehrenberg, 1845, p. 56, 57, 76; Ehrenberg, 1854, pl. 33 (14), fig. 5; pl. 33 (15), fig. 10.

Distephanus stauracanthus (Ehrenberg), Dumitrică, 1973, p. 850, pl. 6 , figs. 14,15 .

\section{Distephanus staurodon (Ehrenberg)}

Dictyocha staurodon Ehrenberg, 1844a, p. 80; Ehrenberg, 1854, pl. 18 , fig. 58.

Dictyocha staurodon Ehrenberg, Locker, 1974, p. 637, pl. 3, fig. 10. Distephanus staurodon (Ehrenberg) Bukry, 1978a, p. 697.

\section{Distephanus sulcatus Bukry}

Distephanus sulcatus Bukry, 1979a, p. 562, pl. 4, figs. 4-12.

Distephanus xenus Bukry, n. sp.

(Plate 1, Figs. 11, 12; Plate 2, Figs. 1-8)

Description: Distephanus xenus has a polygonal basal ring with smooth spineless apices, or apices with only minute, equant, vestigial spines. Prominent basal pikes are longer than the vestigial spines. Totally spineless specimens have rounded apices. The number of apices is typically five to eight, and the sides of the basal ring are slightly concave. The apical structure is a variable complex of polygonal apical openings that are somewhat nonsymmetric.

Remarks: Distephanus xenus is distinguished from Distephanus speculum var. brevispinus Lemmermann by smaller or nonexistent spines and by larger apical ring structures. It is distinguished from Cannopilus tholus Dumitrică by shorter or nonexistent spines and less angular basal-ring apices. The apical structure is larger than either Dictyocha heptacantha Ehrenberg or Cannopilus antarcticus Ciesielski. The combination of large apical structure, typically divided, and nonexistent or extremely small vestigial spines on the basal ring which are shorter than basal-ring pikes helps to distinguish $D$. xenus.

Occurrence: Distephanus xenus occurs in Cores 3 to 5 at DSDP Site 552 in the upper Miocene Dictyocha brevispina Zone and D. fibula Zone. At DSDP Hole 552A it occurs in Cores 22 to 30 assigned to the same zones, in the lower Pliocene and upper Miocene, at similar abundances of 1 to $3 \%$. At DSDP Site 555, also on the Rockall Plateau, it occurs in Cores 5 and 7 assigned to the upper Miocene $D$. brevispina Zone at abundances of 3 to $11 \%$.

Size: Maximum internal diameter 24 to $30 \mu \mathrm{m}$ (holotype $24 \mu \mathrm{m}$ ).

Holotype: USNM 358913 (Plate 1, Figs. 11, 12)

Isotypes: USNM 358914 to 358917 .

Type locality: Rockall Plateau, eastern North Atlantic, DSDP Sample 552-3-2, 123-125 cm.

\section{Distephanus spp.}

Remarks: No specimens of Leg 81 Distephanus with multiply-divided apical rings (cannopilean) were found to have apical pikes (coronatid). Cannopilean or coronatid structural variations can occur in different species of Distephanus which, however, retain their species identity by their ring and spine proportions. For Leg 81 populations of Distephanus it was noted that normal and long-spined forms of $D$. speculum speculum can have cannopilean or coronatid variations, whereas normal and long-spined $D$. speculum minutus has only coronatid variations.

\section{Genus LYRAMULA Hanna, 1928} Lyramula sp.

Remarks: Fragmented specimens, probably derived from Lyramula furcula Hanna, the most common and ubiquitous species of this typical Cretaceous genus. 
Genus MESOCENA Ehrenberg, 1843
Mesocena apiculata apiculata Schulz (Plate 3, Fig. 6)

Mesocena oamaruensis var. apiculata Schulz, 1928, p. 240, fig. 11. Mesocena apiculata (Schulz), Bukry, 1978c, p. 786, pl. 2, fig. 19.

Remarks: Mesocena apiculata apiculata specimens in DSDP Section 406-38-4, are septate but lack spines.

\section{Mesocena apiculata curvata Bukry}

(Plate 3, Fig. 7)

Mesocena apiculata curvata Bukry, 1976b, p. 849, pl. 2, figs. 15, 16.

Mesocena apiculata evexa Bukry, n. subsp.

(Plate 2, Figs. 9-11; Plate 3, Figs. 1-5).

Mesocena apiculata (Schulz), Martini and Müller, 1976 (in part), p. 872, pl. 11, fig. 12 .

Description: Mesocena apiculata evexa has a large, rounded, equilateral, triangular ring with short spines at the apices. The apices are broad and rounded, generally septate, and the sides are moderately convex. A few specimens have one nearly straight side. There are no basal pikes, but very small surface nodes and ornamentation occur.

Remarks: Mesocena apiculata evexa is distinguished from $M$. apiculata apiculata by broad rounded apices, instead of acute apices and by a rounder ring outline because of convex sides. It is distinguished from $M$. apiculata inflata by larger size and relatively shorter spines, from $M$. pappii by broader apices and single apex spines, from $M$. triangula by smaller surface ornamentation and generally septate apices. A few specimens have swirled spines (Plate 3, Fig. 2).

Occurrence: Mesocena apiculata evexa is prominent (1 to 17\%) in the upper Oligocene and lowermost Miocene of DSDP Sites 403 and 406 on the western and southern margins of the Rockall Plateau. It is associated with the acme of diatom Rocella gelida at DSDP 406, and a specimen is recorded with $R$. gelida from DSDP Sample 385-3-3, 60$61 \mathrm{~cm}$ from the western North Atlantic. All the occurrences are in or approximate to the Naviculopsis biapiculata Zone.

Size: Maximum internal diameter 50 to $90 \mu \mathrm{m}$ (holotype $85 \mu \mathrm{m}$ ).

Holotype: USNM 358918 (Plate 3, Fig. 5).

Isotypes: USNM 358919 to 358925 .

Type locality: Rockall Plateau, eastern North Atlantic DSDP Sample 406-30-2, 80-82 cm.

\section{Mesocena apiculata glabra (Schulz)}

Mesocena polymorpha var. triangula Lemmermann fa. glabra Schulz, 1928, p. 237 fig. ?3b, 3c.

Mesocena apiculata glabra (Schulz) Bukry, 1978a, p. 698, pl. 2, figs. $14,15$.

\section{Mesocena circulus (Ehrenberg)}

Dictyocha (Mesocena) circulus Ehrenberg, 1840, p. 208; Ehrenberg, 1854, pl. 19, fig. 44 as Mesocena.

Mesocena circulus (Ehrenberg), Bukry, 1979a, p. 562, pl. 4, figs. 14, 15.

\section{Mesocena diodon nodosa Bukry}

Mesocena diodon nodosa Bukry, 1978b (in part), p. 818, pl. 5, figs. 14,15 ; pl. 6 , figs. $1-3$ (not figs. 4,5 ).

Mesocena diodon nodosa Bukry, Bukry, 1981a, p. 550, pl. 5, fig. 3.

\section{Mesocena oamaruensis Schulz}

Mesocena oamaruensis Schulz, 1928, p. 240, fig. 10a, b.

\section{Mesocena occidentalis Hanna ex Bukry}

Mesocena occidentalis Hanna, 1931, p. 200, pl. E., fig. 1.

Mesocena occidentalis Hanna ex Bukry, Bukry, 1977, p. 834.

\section{Mesocena quadrangula Ehrenberg ex Haeckel .}

Mesocena quadrangula Ehrenberg ex Haeckel, 1887, p. 1556; Lemmermann, 1901, pl. 10, figs. 5-7; fide Loeblich et al., 1968, p. 57. Mesocena quadrangula Ehrenberg ex Haeckel, Bukry, 1980, p. 553, pl. 7, figs. 9-17; pl. 8, figs. 1-10.

\section{Mesocena triangula (Ehrenberg)}

Dictyocha triangula Ehrenberg, 1839, p. 139; Ehrenberg, 1854, pl. 22, figs. 41 as Mesocena.

Mesocena triangula (Ehrenberg) Ehrenberg, Bukry et Foster, 1973, p. 829 , pl. 6 , figs. $9-10$.

\section{Mesocena triodon Bukry}

Mesocena triodon Bukry, 1973a, p. 860, pl. 2, fig. 11.

\section{Mesocena sp.}

Remarks: Fragmented, obscured, indeterminate specimens are tabulated in this category.

\section{Genus NAVICULOPSIS Frenguelli, 1940 Naviculopsis aspera (Schulz)}

Dictyocha navicula var. aspera Schulz, 1928, p. 246, fig. 20a, b. Naviculopsis aspera (Schulz), Bukry, 1976a, p. 896, pl. 8, figs. 11, 12. Naviculopsis aspera (Schulz) Perch-Nielsen, 1976, p. 35, figs. 8, 9, 31, 34,35 .

\section{Naviculopsis biapiculata (Lemmermann)}

Dictyocha navicula var. biapiculata Lemmermann, 1901, p. 258, pl. 10 , figs. 14?, 15 .

Naviculopsis biapiculata (Lemmermann), Ling, 1972 (in part), p. 181, pl. 30, figs. 1, 2 (not figs. 3, 4).

Naviculopsis biapiculata (Lemmermann), Bukry, 1975b (in part), p. 856 , pl. 6 , figs. $5,7,8$ (not fig. 6 ).

Remarks: Some specimens of Naviculopsis biapiculata in DSDP Section 406-32-3, have curved spines.

\section{Naviculopsis constricta (Schulz) Bukry emend.}

Dictyocha navicula var. biapiculata fa. constricta Schulz, 1928, p. 246, fig. 21.

Naviculopsis constricta (Schulz) Bukry emend., in Barron, Bukry, and Poore, in press.

\section{Naviculopsis eobiapiculata Bukry}

Naviculopsis eobiapiculata Bukry, 1978c, p. 787, pl. 4, figs. 9-16.

\section{Naviculopsis foliacea Deflandre}

Naviculopsis foliacea Deflandre, 1950, p. 204, figs. 235-240.

\section{Naviculopsis lata (Deflandre)}

Dictyocha biapiculata var. lata Deflandre, 1932, p. 500, figs. $30,31$. Naviculopsis lata (Deflandre), Bukry, 1982a, p. 431, pl. 7, figs. 11-14.

\section{Naviculopsis minor (Schulz)}

Dictyocha navicula E. var. minor n. v. Schulz, 1928, p. 246, fig. 22. Naviculopsis minor (Schulz 1928) Frenguelli 1940, Martini, 1981, p. 282 , pl. 1 , figs. $8,9 \mathrm{a}, \mathrm{b}$.

Naviculopsis minor (Schulz) Bukry, in Barron, Bukry, and Poore, in press.

Naviculopsis pacifica pacifica (Dumitrică) Bukry, n. comb.

Naviculopsis quadrata ssp. pacifica Dumitrică, 1973, p. 846, pl. 1, figs. 12-14.

Remarks: Naviculopsis quadrata (Ehrenberg) (see Loeblich et al. 1968; Ling, 1973; and Locker, 1974) has short pointed conical spines, and broad, nearly straight ring ends. The spine is less than half the ring width (Bukry, 1978a). Naviculopsis pacifica pacifica (Dumitrica) is distinguished by long, cylindrical spines with rounded ends, and more curved ring ends. Similar long cylindrical spines with round ends are unusual, but occurred in Eocene Mesocena occidentalis Hanna ex Bukry, suggesting the change is a response to an unusual environmental factor. Spine form appears to have been genetically conservative in the silicoflagellate record.

Naviculopsis pacifica pacifica occurs commonly in DSDP Sample 206-35, CC, which is assigned to coccolith Zone CN1a, very near the Oligocene/Miocene boundary, with diatom Rocella gelida (see Dumitricà, 1973, and Bukry, 1973b). 
Naviculopsis pacifica pansa Bukry, n. subsp.

(Plate 1, Figs. 8-10)

Naviculopsis quadrata (Ehrenberg), Bukry, 1978b (in part), p. 706, pl. 3 , figs. 7,8 .

Description: Naviculopsis pacifica pansa has an oblong ring with an apical bar along the minor axis and two spines which flare and end bluntly, instead of in points. The unusual spines are longer than half of the minor axis length. In width, the spines, bar, and ring are about equal. The minor axis equals about $40 \%$ of the major axis length.

Remarks: Naviculopsis pacifica pansa is distinguished from N. pacifica pacifica by the flaring of the spines, their blunt termination, and the slightly more quadrate ring shape. It is distinguished from Naviculopsis quadrata by the long spines and their flared, blunt terminations. The younger, Miocene, minor genus, Deflandryocha (Jerković, 1963) has flared spines similar to N. pacifica pansa.

Occurrence: Naviculopsis pacifica pansa occurs in Cores 29 and 30 of DSDP Site 406, at or slightly above the Oligocene/Miocene boundary on Rockall Plateau. It has also been illustrated from Core 13 of DSDP Hole 369A off northwestern Africa. Both occurrences were assigned to uppermost Oligocene Zone CP19 or CN1a by coccoliths, indicating potential usefulness for locating the Miocene/Oligocene boundary in the North Atlantic.

Size: Maximum internal length 40 to $60 \mu \mathrm{m}$ (holotype $60 \mu \mathrm{m}$ ).

Holotype: USNM 358910 (Plate 1, Fig. 8).

Isotypes: USNM 358911 and 358912.

Type locality: Rockall Plateau, eastern North Atlantic Ocean, DSDP Sample 406-30-2, 80-82 cm.

\section{Naviculopsis sp.}

Remarks: Indeterminate or obscured specimens are assigned to this category.

\section{Incertae Sedis Genus MACRORA Hanna, 1932 Macrora stella (Azpeitia)}

Pyxidicula(??) stella Azpeitia, 1911, p. 150, 152, 213, pl. 1, fig. 1. Macrora stella (Azpeita) Hanna, 1932, p. 196, pl. 12, fig. 7. Macrora stella (Azpeita), Bukry, 1978c, p. 788, pl. 4, figs. 20, 21.

\section{ACKNOWLEDGMENTS}

I thank Gerta Keller and John A. Barron, U.S. Geological Survey, for their constructive reviews of this manuscript. Some supplementary slides from DSDP Legs 48 and 81 were provided by Jack G. Baldauf and John A. Barron, U.S. Geological Survey. Copies of revised site chapters were made available by DSDP Leg $81 \mathrm{Co}$-Chief Scientist David G. Roberts and Scientific Representative John B. Keene. I also thank Dorothy L. Blackstock, U.S. Geological Survey, for proficient proofing and typing of the text and figures. Samples from DSDP Leg 48 were provided by the National Science Foundation Deep Sea Drilling Project.

\section{REFERENCES}

Azpeitia, D. F. M., 1911. La Diatomologia Española en los Comienzos des Siglo XX. Assoc. Esp. Prog. Cien. Congr. Zargoza, 4(2): $1-320$.

Barron, J. A., 1976. Revised Miocene and Pliocene diatom biostratigraphy of upper Newport Bay, Newport Beach, California. Mar. Micropaleontol. 1:27-63.

1980. Lower Miocene to Quaternary diatom biostratigraphy of Leg 57, off northeastern Japan, Deep Sea Drilling Project. In Langseth, M., Okada, H., von Huene, R., Nasu, N., et al., Init. Repts. DSDP, 56, 57, Pt. 2: Washington (U.S. Govt. Printing Office), 641-685.

Barron, J. A., Bukry, D., and Poore, R. Z., in press. Correlation of the middle Eocene Kellogg shale of northern California. Micropaleontology.

Bukry, D., 1973a. Coccolith and silicoflagellate stratigraphy, Deep Sea Drilling Project Leg 18, eastern North Pacific. In Kulm, L. D., von Huene, R., et al., Init. Repts. DSDP, 18: Washington (U.S. Govt. Printing Office), 817-831.

1973b. Coccolith and silicoflagellate stratigraphy, Tasman Sea and southwestern Pacific Ocean, Deep Sea Drilling Project Leg 21. In Burns, R. E., Andrews, J. E., et al., Init. Repts. DSDP. 21: Washington (U.S. Govt. Printing Office), 885-893. 1975a. Coccolith and silicoflagellate stratigraphy, northwestern Pacific Ocean, Deep Sea Drilling Project Leg 32. In Larson, R. L., Moberly, R., et al., Init. Repts. DSDP, 32: Washington (U.S. Govt. Printing Office), 677-701.

1975b. Silicoflagellate and coccolith stratigraphy, Deep Sea Drilling Project Leg 29. In Kennett, J. P., Houtz, R. E., et al., Init. Repts. DSDP, 29: Washington (U.S. Govt. Printing Office), 845-872.

1976a. Cenozoic silicoflagellate and coccolith stratigraphy, South Atlantic Ocean, Deep Sea Drilling Project Leg 36. In Hollister, C. D., Craddock, C., et al., Init. Repts. DSDP, 35: Washington (U.S. Govt. Printing Office), 885-917.

1976b. Silicoflagellate and coccolith stratigraphy, Norwegian-Greenland Sea, Deep Drilling Project Leg 38. In Talwani, M., Udintsev, G., et al., Init. Repts. DSDP, 38: Washington (U.S. Govt. Printing Office), 843-855.

1976c. Silicoflagellate and coccolith stratigraphy, southeastern Pacific Ocean, Deep Sea Drilling Project Leg 34. In Yeats, R. S., Hart, S. R., et al., Init. Repts. DSDP, 34: Washington (U.S. Govt. Printing Office), 715-735.

1977. Coccolith and silicoflagellate stratigraphy, South Atlantic Ocean, Deep Sea Drilling Project Leg 39. In Supko, P. R. Perch-Nielsen, K., et al., Init. Repts. DSDP, 39: Washington (U.S. Govt. Printing Office), 825-839.

1978a. Cenozoic coccolith and silicoflagellate stratigraphy, offshore northwest Africa, Deep Sea Drilling Project Leg 41. In Lancelot, Y., Seibold, E., et al., Init. Repts. DSDP, 41: Washington (U.S. Govt. Printing Office), 689-707.

1978b. Cenozoic coccolith, silicoflagellate, and diatom stratigraphy, Deep Sea Drilling Project Leg 44. In Benson, W. E., Sheridan, R. E., et al., Init. Repts. DSDP, 44: Washington (U.S. Govt. Printing Office), 807-863.

1978c. Cenozoic silicoflagellate and coccolith stratigraphy, northwestern Atlantic Ocean, Deep Sea Drilling Project Leg 43. In Benson, W. E., Sheridan, R. E., et al., Init. Repts. DSDP, 44: Washington (U.S. Govt. Printing Office), 775-805.

1979a. Coccolith and silicoflagellate stratigraphy, northern Mid-Atlantic Ridge and Reykjanes Ridge, Deep Sea Drilling Project Leg 49. In Luyendyk, B. P., Cann, J. R., et al., Init. Repts. DSDP, 49: Washington (U.S. Govt. Printing Office), 551-581. 1979b. Comments on opal phytoliths and stratigraphy of Neogene silicoflagellates and coccoliths at Deep Sea Drilling Project Site 397 off northwest Africa. In Luyendyk, B. P., Cann, J. R. et al., Init. Repts. DSDP, 49: Washington (U.S. Govt. Printing Office), 977-1009.

1980. Silicoflagellate biostratigraphy and paleoecology in the eastern equatorial Pacific, Deep Sea Drilling Project Leg 54. In Hekinian, R., Rosendahl, B. R., et al., Init. Repts. DSDP, 54: Washington (U.S. Govt. Printing Office), 545-573. 1981a. Silicoflagellate stratigraphy of offshore California and Baja California, Deep Sea Drilling Project Leg 63. In Yeats, R. S., Haq, B. U., et al., Init. Repts. DSDP, 63: Washington (U.S. Govt. Printing Office), 539-557.

1981b. Synthesis of silicoflagellate stratigraphy for Maestrichtian to Quaternary marine sediment. Soc. Econ. Paleontol. Mineral. Spec. Publ., 32:433-444.

1982a. Cenozoic silicoflagellates from offshore Guatemala, Deep Sea Drilling Project Site 495. In von Huene, R., Auboin, J., et al., Init. Repts. DSDP, 67: Washington (U.S. Govt. Printing Office), 425-445.

1982b. Neogene silicoflagellates of the eastern Equatorial Pacific, Deep Sea Drilling Project Hole 503A. In Prell, W. L. Gardner, J. V., et al., Init. Repts. DSDP, 68: Washington (U.S. Govt. Printing Office), 311-323.

1983. Upper Cenozoic silicoflagellates from offshore Ecuador, Deep Sea Drilling Project Site 504. In Cann, J. R., Langseth, M. G., et al., Init. Repts. DSDP, 69: Washington (U.S. Govt. Printing Office), 321-342.

Bukry, D., and Foster, J. H., 1973. Silicoflagellate and diatom stratigraphy, Leg 16, Deep Sea Drilling Project. In van Andel, Tj. H., Heath, G. R., et al., Init. Repts. DSDP, 16: Washington (U.S. Govt. Printing Office), 815-871.

Deflandre, G., 1932. Sur la systématique des Silicoflagellés. Soc. Bot. France Bull., 79:494-506. 
, 1950. Contribution a l'étude des silicoflagellidés actuels et fossiles. Microscopie, 2:72-108, 117-142, and 191-210.

Dumitrič, P., 1973. Paleocene, late Oligocene and post-Oligocene silicoflagellates in southwestern Pacific sediments cored on DSDP Leg 21. In Burns, R. E., Andrews, J. E., et al., Init. Repts. DSDP, 21: Washington (U.S. Govt. Printing Office), 837-883.

1978. Badenian silicoflagellates from Central Paratethys. In Brestenská, E. (Ed.), Chronostratigraphie und Neostratotypen, Miozän der Zantralen Paratethys (Vol. 6): Bratislava (Veda), pp. 207-230.

Dzinoridze, R. N., Jousé, A. P., Koroleva-Golikova, G. S., Kozlova, G. E., Nagaeva, G. S., Petrushevskaya, M. G., and Strelnikova, N. I., 1979. Diatom and radiolarian Cenozoic stratigraphy, Norwegian Basin; DSDP Leg 38. In Talwani, M., Udintsev, G., et al., Init. Repts. DSDP, Suppl. to Vols. 38, 39, 40, and 41: Washington (U.S. Govt. Printing Office), 289-427.

Ehrenberg, C. G., 1839. Über die Bildung der Kreidefelsen und des Kreidemergels durch unsichtbare Organismen. K. Preuss. Akad. Wiss. Berlin Ber., Jahrg. 1838:59-148.

1840. 274 Blätter von ihm selbst ausgeführter Zeichnungen von ebenso vielen Arten. K. Preuss. Akad. Wiss. Berlin Ber., Jahrg. 1840:197-219.

, 1844a. Mittheilung über zwei neue Lager von Gebirgsmassen aus Infursorien als Meeres-Absatz in Nord-Amerika und eine Vergleichung derselben mit den organischen Kreide-Gebilden in Europa und Afrika. K. Preuss. Akad. Wiss. Berlin Ber., Jahrg. 1844:57-97.

1844b. Untersuchungen über die kleinsten Lebensformen im Quellenlande des Euphrats und Araxes, so wie über eine an neuen Formen sehr reiche marine Tripelbildung von den BermudaInseln vor. K. Preuss. Akad. Wiss. Berlin Ber., Jahrg. 1844: 253-275.

1845. Neue Untersuchungen über das kleinste Leben als geologisches Moment. K. Preuss. Akad. Wiss. Berlin Ber., Jahrg. 1845:53-87.

1854. Mikrogeologie: Leipzig (Leopold Voss), 1-374.

Frenguelli, J., 1940. Consideraciones sobre los sílicoflagelados fosiles. Mus. La Plata Rev. Paleontol., 2(7):37-112.

1951. Silicoflagelados del Tripoli de Mejillones (Chile). Physis (Buenos Aires), 20:272-284.

Glezer, Z. I., 1962. K voprosu o filogeneze kremnevykh zhgutikovykh vodorosley [On the phylogeny of silicoflagellates]. Paleont. Zhur. 1962(1):146-156.

1966. Silicoflagellatophyceae. In Gollerbakh, M. M. (Ed.) Cryptogamic plants of the U.S.S.R. Akad. Nauk SSSR, V. A. Komarova Bot. Inst. (Translated from Russian by Israel Program for Scientific Translations Lts., Jerusualem, 1970), 7:1-363.

Haeckel, E. H. P. A., 1887. Cannorrhaphida. Challenger Rept., 18: 1546-1569.

Hanna, G. D., 1931. Diatoms and silicoflagellates of the Kreyenhagen shale. Calif. Div. Mines Geol. State Mineral. Rept., 27:187-201.

1932. The diatoms of Sharktooth Hill, Kern County, California. Calif. Acad. Sci. Proc., 20:161-263.
Ichikawa, W., Shimizu, I., and Bachmann, A., 1967. Fossil silicoflagellates and their associated uncertain forms in Iida Diatomite, Noto Peninsula, Central Japan. Kanazawa Univ. Sci. Repts., 12: 143-172.

Jerković, L., 1963. Sur un nouveau type de silicoflagellidé fossile, $D e-$ flandryocha nov. gen., à cornes radiales spatulées. C. R. Acad. Sci. 256:2202-2204.

1965. Sur quelques silicoflagellidés de Yougoslavie. Lab. Micropaleont., Inst. Paleont. Museum Paris, 1-8.

Kaneps, A. G., 1979. Gulf Stream: Velocity fluctuations during the Late Cenozoic. Science, 204:297-301.

Keigwin, L. D., Jr., 1979. Late Cenozoic stable isotope stratigraphy and paleoceanography of DSDP sites from the east equatorial and central North Pacific Ocean. Earth Planet. Sci. Lett., 45:361-382.

Kitchell, J. A., 1979. Paleocene paleoproductivity in the central Arctic: Evidence from siliceous phytoplankton. Geol. Soc. Am. Abstract with Programs, 11:458. (Abstract)

Lemmermann, E., 1901. Silicoflagellatae. Deutsche. Bot. Gesell. Ber., 19:247-271.

Ling, H. Y., 1972. Upper Cretaceous and Cenozoic silicoflagellates and ebridians. Am. Paleontol. Bull., 62:135-229.

1973. Silicoflagellates and ebridians from Leg 19. In Creager, J. S., Scholl, D. W., et al., Init. Repts. DSDP, 19: Washington (U.S. Govt. Printing Office), 751-775.

Locker, S., 1974. Revision der Silicoflagellaten aus der Mikrogeologischen Sammlung von C. G., Ehrenberg. Eclogae Geol. Helv., 67 631-646.

1975. Dictyocha varia sp. n., eine miozäne Silicoflagellaten-Art mit kompliziertem Variationsmodus. Z. Geol. Wiss., Berlin, 3:99-103.

Loeblich, A. R., III, Loeblich, L. A., Tappan, H., and Loeblich, A. R., Jr., 1968. Annotated index of fossil and recent silicoflagellates and ebridians with descriptions and illustrations of validly proposed taxa. Geol. Soc. Am. Mem., 106:1-319.

Martini, E., 1981. Silicoflagellatem im Paläogen von Norddeutschland. Senckenb. Lethaea, 62:277-283.

Martini, E., and Müller, C., 1976. Eocene to Pleistocene silicoflagellates from the Norwegian-Greenland Sea (DSDP Leg 38). In Talwani, M., Udintsev, G., et al., Init. Repts. DSDP, 38: Washington (U.S. Govt. Printing Office), 857-895.

Perch-Nielsen, K., 1975. Late Cretaceous to Pleistocene silicoflagellates from the southern southwest Pacific, DSDP Leg 29. In Kennett, J. P., Houtz, R. E., et al., Init. Repts. DSDP, 29: Washington (U.S. Govt. Printing Office), 677-721.

1976. New silicoflagellates and a silicoflagellate zonation in north European Palaeocene and Eocene diatomites. Geol. Soc. Denmark Bull., 25:27-40.

Schulz, P., 1928. Beiträge zur Kenntnis fossiler und rezenter Silicoflagellaten. Bot. Archiv, 21:225-292.

Stradner, H., 1961. Über fossile Silicoflagelliden und die Möglichkeit ihrer Verwendugn in der Erdölstratigraphie. Erdöl Kohle, 14:87-92.

Date of Acceptance: August 11, 1983 

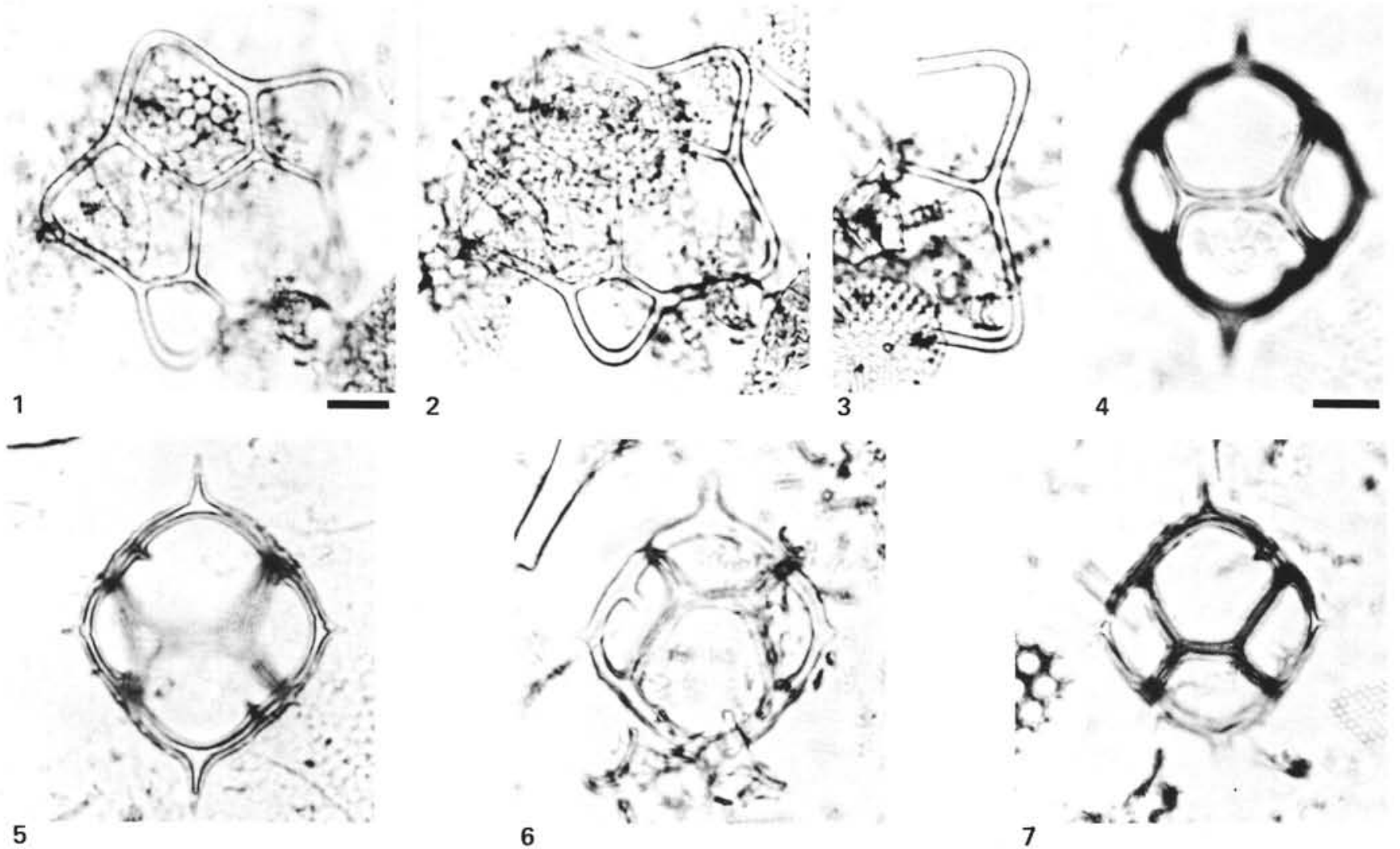

5
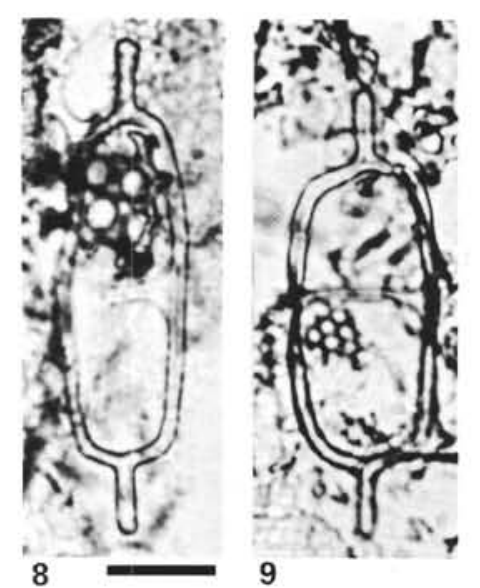

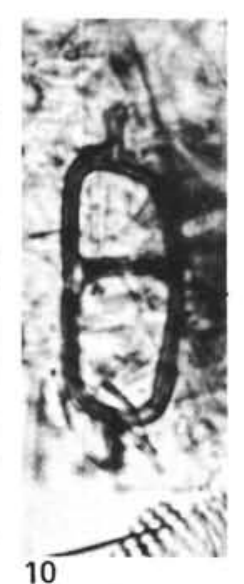

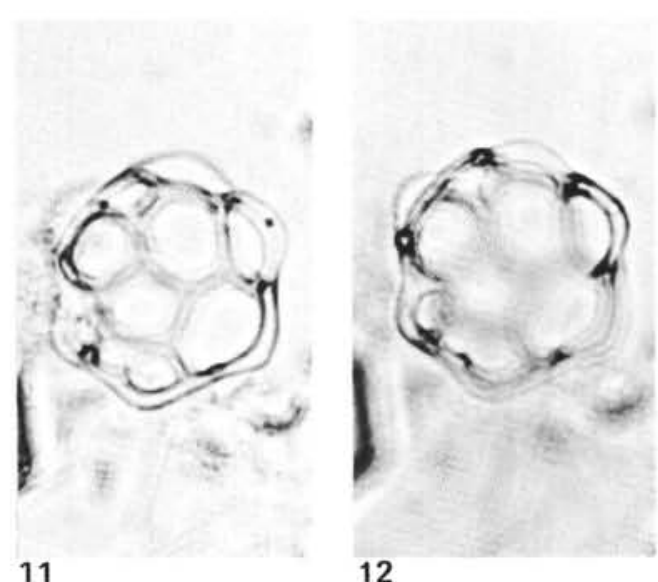

11
12

Plate 1. Silicoflagellates from the Rockall Plateau. (Scale bar for Figs. 1-3 equals $20 \mu \mathrm{m}$, for Figs. 4-7, 11,12 equals $10 \mu \mathrm{m}$, and for Figs $8-10$ equals $20 \mu \mathrm{m}$.) 1-3. Corbisema panda Bukry, n. sp. DSDP Sample 553A-10-1, 140-142 cm. $(1,2)$ Holotype, USNM 358905, high and low focus; (3) USNM 358906, fragment. 4-7. Dictyocha pulchella Bukry var. inflata Bukry, n. var. (4, 5) Holotype, USNM 358907, DSDP Sample 555-18-2, 73-75 cm, high and low focus; (6) USNM 358908, DSDP Sample 555-19-2, 80-82 cm; (7) USNM 358909, DSDP Sample 555-21-2, 80-82 cm. 8-10. Naviculopsis pacifica Dumitricǎ subsp. pansa Bukry, n. subsp. DSDP Sample 406-30-2, 80-82 cm. (8) Holotype, USNM 358910; (9) USNM 358911; (10) USNM 358912. 11, 12. Distephanus xenus Bukry, n. sp. Sample 552-3-2, 123-125 cm. Holotype, USNM 358913 , high and low focus. 

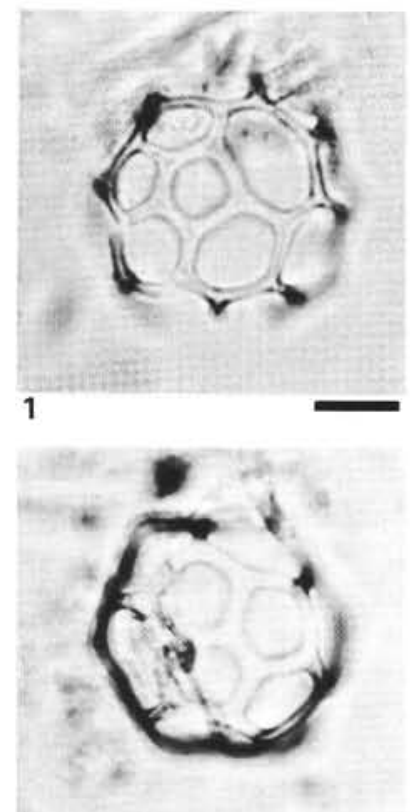

5

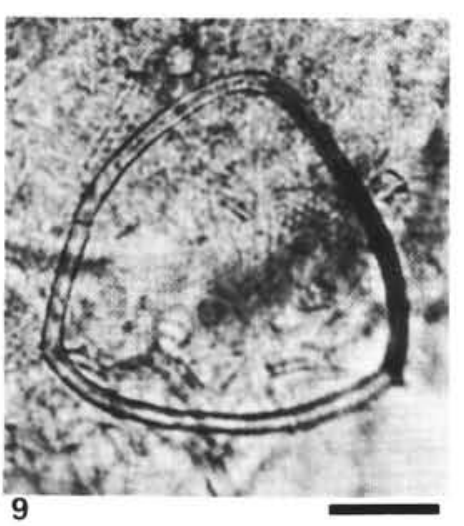

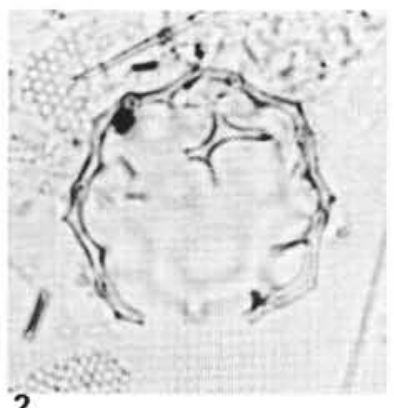

2

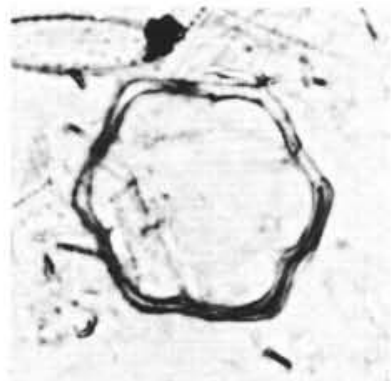

6

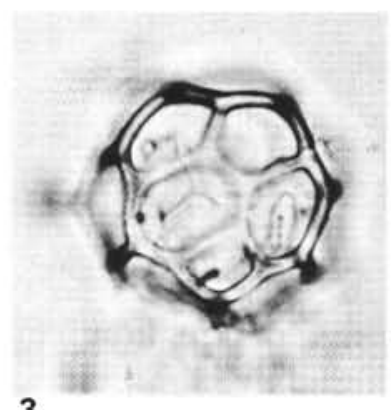

3

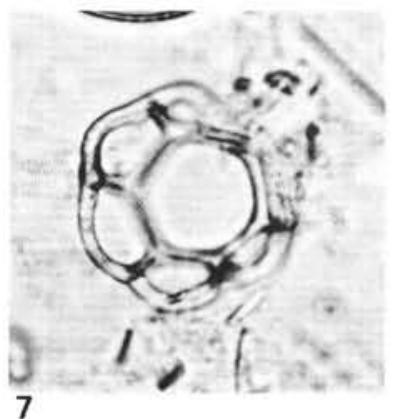

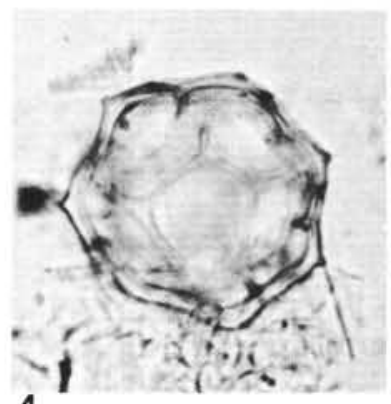

4

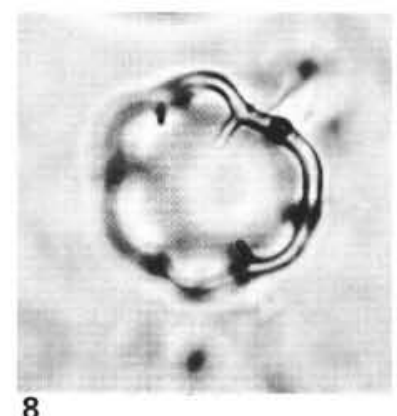

8

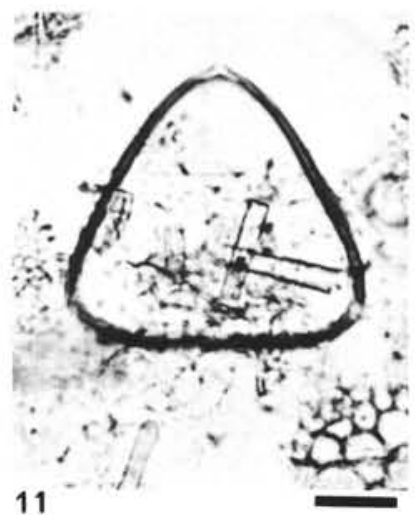

Plate 2. Silicoflagellates from Rockall Plateau. (Scale bar for Figs. 1-8 equals $10 \mu \mathrm{m}$, for Figs. 9 and 10 equals $20 \mu \mathrm{m}$, and for Fig. 11 equals 20 $\mu \mathrm{m}$.) 1-8. Distephanus xenus Bukry, n. sp. (1, 2) USNM 358914, DSDP Sample 552-4-3, 130-132 cm, high and low focus; $(3,4)$ USNM 358915 , DSDP Sample 552-4-3, 130-132 cm; $(5,6)$ USNM 358916, DSDP Sample 552-3-2, 123-125 cm; (7, 8) USNM 358917, DSDP Sample 552-3-2, 123-125 cm. 9-11. Mesocena apiculata (Schulz) subsp. evexa Bukry, n. subsp. (9) USNM 358919, DSDP Sample 406-25-1, 70-72 cm; (10) USNM 358920, DSDP Sample 406-29-1, 30-32 cm; (11) USNM 358921, DSDP Sample 406-33-1, 90-91 cm. 

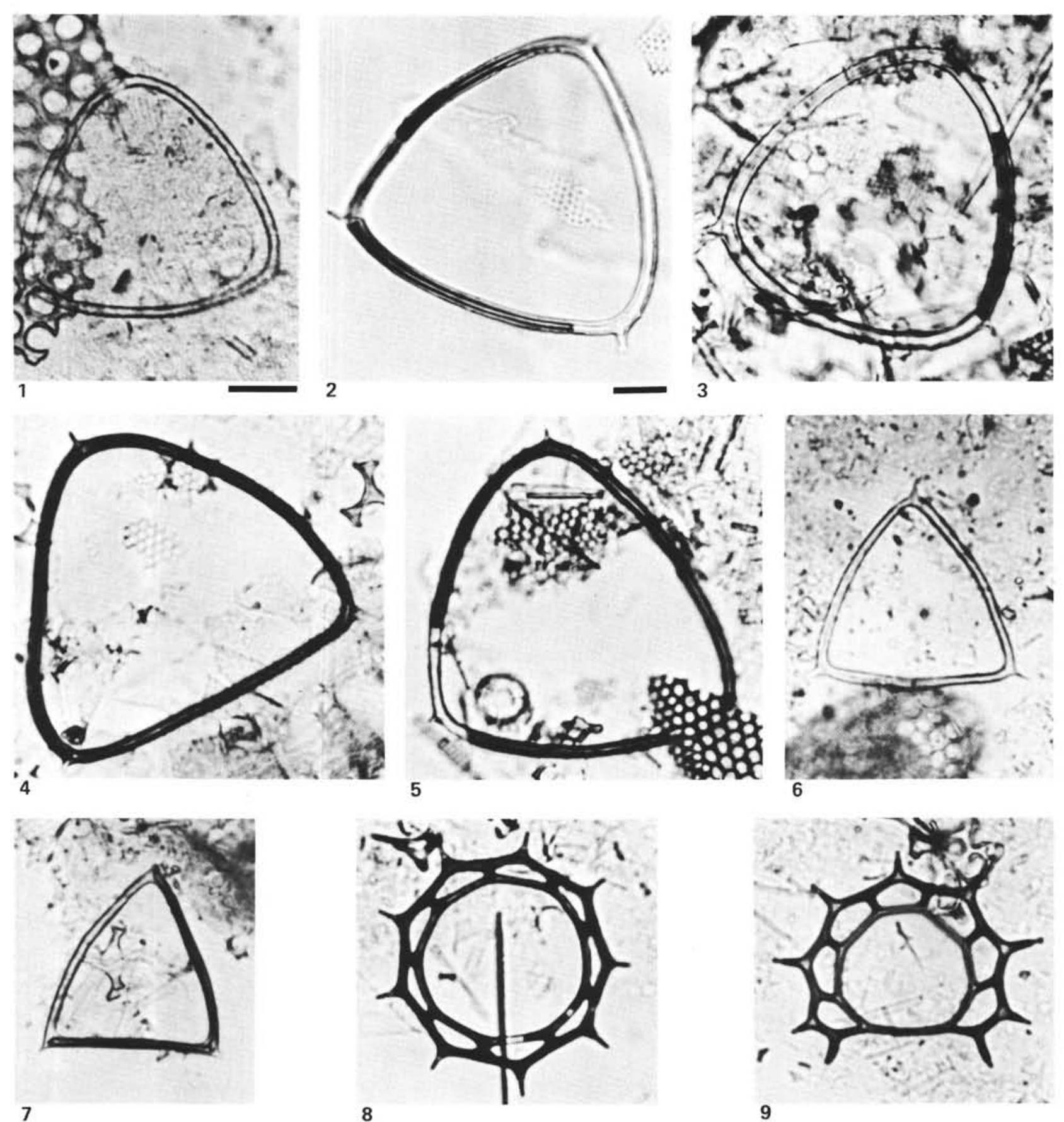

Plate 3. Silicoflagellates from Rockall Plateau. (Scale bar for Figs. 1, 3-9 equals $20 \mu \mathrm{m}$, and for Fig. 2 equals $10 \mu \mathrm{m}$.) 1-5. Mesocena apiculata (Schulz) subsp. evexa Bukry, n. subsp. (1) USNM 358922, DSDP Sample 406-27-1, 30-31 cm; (2) USNM 358923, DSDP Sample 406-25-1, 70-72 cm; (3) USNM 358924, DSDP Sample 406-30-2, 80-82 cm; (4) USNM 358925, DSDP Sample 406-31-1, 105-105 cm; (5) Holotype, USNM 358918 , DSDP Sample 406-30-2, 80-82 cm. 6. Mesocena apiculata apiculata Schulz. DSDP Sample 403-26-2, 50-52 cm. 7. Mesocena apiculata curvata Bukry. DSDP Sample 406-30-2, 80-82 cm. 8, 9. Distephanus polyactis (Ehrenberg) DSDP Sample 552A-18-1, 95-97 cm. (8) Normal; (9) Incomplete symmetry. 